Chimia 46 (1992)186-199

(1) Neue Schweizerische Chemische Gesellschaft ISSN 0009-4293

\title{
Instrumentelle Methoden der anorganischen Elementanalytik
}

\author{
Niklaus Kläntschi*
}

Abstract. Instrumental element analysis has developed enormously during the past decades. It has rightfully assumed a major role in the analytical laboratory in the field of materials science, environmental analysis, monitoring and research. Due to its high accuracy, classical chemical analysis is still most useful for elemental determinations at major levels and for special applications. In a modern inorganic analytical laboratory, a variety of different instrumental techniques is employed for qualitative and quantitative analysis, covering practically all the elements of the periodic system in a variety of different matrices, from major to trace and ultratrace mass levels. The prerequisite for atomic spectroscopy is the step of excitation, normally in combination with a process of atomization. From a number of different atomization processes currently used, inductively coupled plasma (ICP) is now the most prominent. The atomic spectrometric methods commonly employed in an analytical laboratory are described. These include atomic absorption, both with flame and graphite furnace, ICP atomic emission and ICP mass spectrometry, X-ray fluorescence together with ion chromatography as a nonspectrometric analytical process. The different instrumental techniques are critically compared in relation to number of detectable elements and species, limits of detection, precision, sample throughput, dynamic concentration range, degree of automation, and cost of equipment. Moreover, the importance of sampling and sample treatment prior to the analytical determination, quality assurance, and mode of automation are related both to the present situation and future trends in instrumental element analysis.

\section{Einleitung}

'Analytical chemistry is the science of chemical measurements. It is fundamentally an experimental science' [1].

Als Folge des apparativen und technologischen Fortschritts befindet sich die chemische Analytik in rascher Entwicklung. Die Ansprüche an Materialqualitäten steigen ständig. Das zunehmende Bewusstsein und die wissenschaftliche Bearbeitung toxikologisch und ökologisch relevanter Fragestellungen bescheren der Analytik neue Aufgaben und stellen höhere Anforderungen an sie. Rund $20 \%$ aller Chemiker sind weltweit in der chemischen Analytik oder verwandten Gebieten tätig. Der jährliche Gesamtumsatz analytischer Instrumentenhersteller entspricht einem Milliardendollarmarkt mit jährlichen Zuwachsraten von 10\% [2].

Die anorganische Instrumentalanalytik befasst sich mit der Bestimmung von Elementen und Verbindungen der Atome $\mathrm{H}$ (Wasserstoff) bis U (Uran). Ausgenom-

*Korrespondenz: Dr. N. Kläntsch EMPA, Eidg. Materialprüfungs- und Forschungsanstalt

Überlandstrasse 129

CH-8600 Dübendorf men sind Edelgase und die Stoffgruppe derorganischen Kohlenstoffverbindungen, mit deren Charakterisierung und Quantifizierung sich die organische Analytik befasst. Isotopenanalysen sollen hier lediglich am Rand erwähnt werden. Sie können

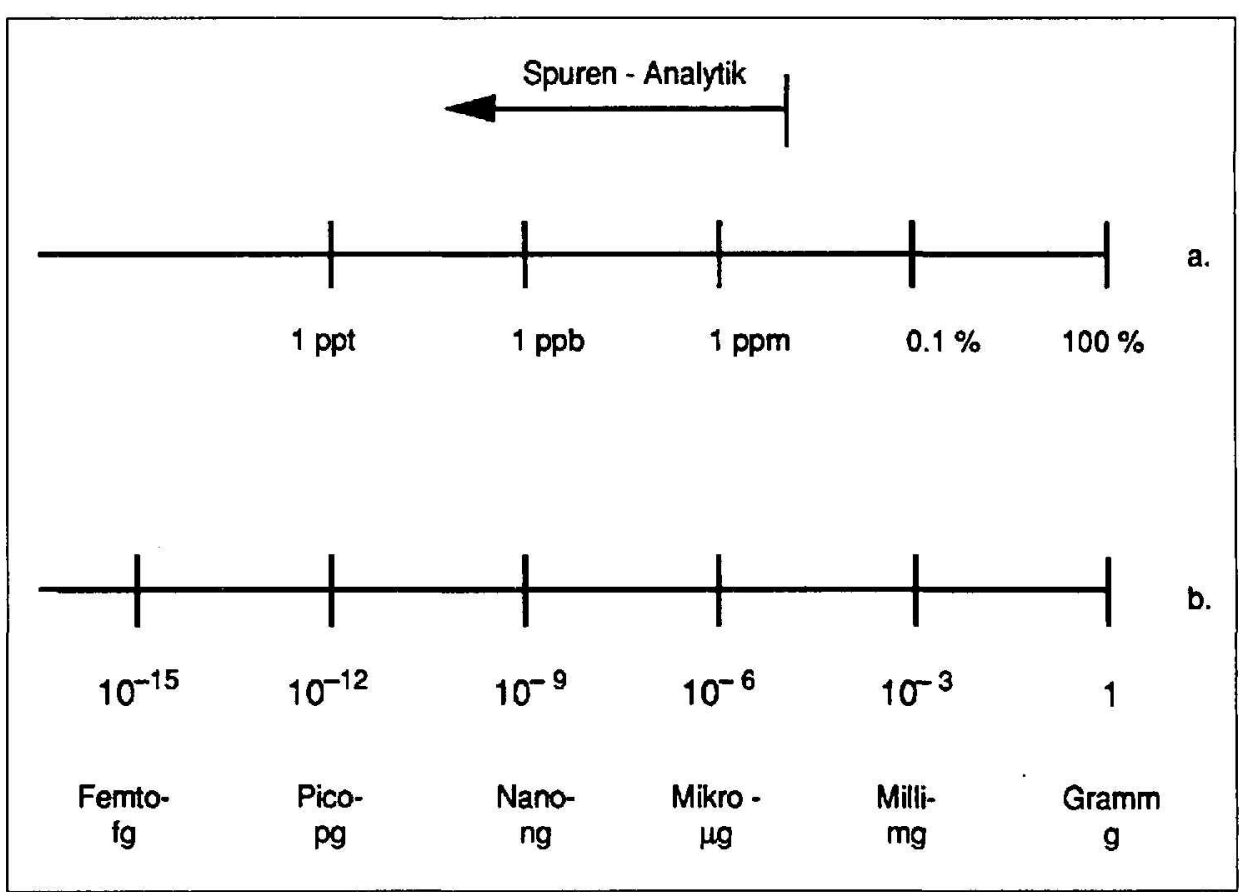

Fig. 1. Bereich der Elementanalyse; a) Massenanteile; b) Massen absolut
Niklaus Kläntschi, 1942 geboren in Bern. 1962-66 Studium an der Abteilung für Naturwissenschaften an der ETH-Zürich, Diplom in anorg. Chemie. Die Promotionsarbeit unter Leitung von Prof. W. Schneider (ETH-Zürich) zum Thema kinetischer Untersuchungen zum Einbau von Eisen in Porphyrine wurde 1970 abgeschlossen. Anschliessend folgte ein Aufenthalt an der Stanford University, Palo Alto, USA bei Prof, E.E. van Tamelen mit Arbeiten zu Reaktionsmechanismen an Titancyclopentadienyl-Komplexen in nichtwässr. Medien. Zurück in der Schweiz schloss sich eine mehrjährige Tätigkeit als F+E-Chemiker in der Industrie an mit nachfolgendem Übertritt an die EMPA-Dübendorf. Intensive Tätigkeit in anorg. Instrumentalanalytik, 1986 Übernahme der Leitung der Abteilung Anorg. Chemie, seit 1990 Leitung des Ressorts Chemie an der EMPA-Dübendorf. Lehrauftrag der ETH-Zürich und Universität Zürich.

in gewissen Fällen wichtige Informationen liefern, z.B. in Fragen der Zuordnung oder Herkunft eines untersuchten Materials.

Der Begriff instrumentelle Methoden versteht sich als Abgrenzung zu den nass- 
Tab. 1. Beispiele untersuchter Objekte

\begin{tabular}{|c|c|c|c|}
\hline $\begin{array}{l}\text { Art des } \\
\text { Untersuchungsobjektes }\end{array}$ & $\begin{array}{l}\text { Aggregat- } \\
\text { Zustand }\end{array}$ & Beispiel aus der Praxis & Analytische Relevanz \\
\hline metallische Werkstoffe & fest & Stahl, Leichtmetalle, Legierungen aller Art & Qualitätssicherung. Sicherheit, Schadenfalle \\
\hline nichtmetallische Werkstoffe & fest & $\begin{array}{l}\text { Keramik, Kunststoff, Gummi, Glas, } \\
\text { Verbund-Werkstoffe, Holz }\end{array}$ & Schadenfallle, Toxikologie, Forschung \\
\hline $\begin{array}{l}\text { Industrie- und Forschungs- } \\
\text { Produkte }\end{array}$ & flüssig, fest & $\begin{array}{l}\text { Reinstmetalle und-Legierungen, Keramik-, } \\
\text { Hochtemperaturwerkstoffe, Industriediamanten } \\
\text { Pharmazeutische Produkte }\end{array}$ & Qualitätssicherung, Forschung \\
\hline $\begin{array}{l}\text { gewerbliche Produkte, } \\
\text { Baustoffe }\end{array}$ & flüssig, fest & $\begin{array}{l}\text { Anstrichstoffe, Sportplatzbeläge } \\
\text { und-Markierungen, Wärme- } \\
\text { dämmungsmaterial, Holz }\end{array}$ & $\begin{array}{l}\text { Toxikologie, Ökologie, Schadenfälle. } \\
\text { Sicherheit, Forschung }\end{array}$ \\
\hline Publikumsprodukte & flüssig, fest & $\begin{array}{l}\text { Spraydosen, Batterien, Zahnbürsten, } \\
\text { Skischuhe, Kehrrichtsäcke }\end{array}$ & Toxikologie, Ökologie, Schadenfälle \\
\hline Monitorproben/Überwachung & flïssig, fest & $\begin{array}{l}\text { Wasser-, Staub-, und Filterproben, } \\
\text { Absorptionslösungen, Boden- und Grasproben }\end{array}$ & Toxikologie, Ökologie \\
\hline Rückstănde & flüssig, fest & $\begin{array}{l}\text { Korrosionsprodukte, Ablagerungen, Rückstände, } \\
\text { Aschen }\end{array}$ & Schadenfälle, Sicherheit \\
\hline $\begin{array}{l}\text { Abfallprodukte, Produkte } \\
\text { der Entsorgung }\end{array}$ & flüssig, fest & $\begin{array}{l}\text { Sickerwasser, Aschen und Schlacken aus } \\
\text { Kehrrichtverbrennungsanlagen und Rauchgas- } \\
\text { reinigung, Rückstände aus Produktionsprozessen, } \\
\text { Sonderabfall }\end{array}$ & $\begin{array}{l}\text { Toxikologie, Ökologie, Identifizierung, } \\
\text { Forschung }\end{array}$ \\
\hline Gesteine, Mineralien & fest & $\begin{array}{l}\text { Boden- und Gesteinsproben, geologiesche } \\
\text { Exploration, Erze }\end{array}$ & $\begin{array}{l}\text { Forschung, Identifizierung. Frage der } \\
\text { Herkunft }\end{array}$ \\
\hline Kunst, Schmuck & fest & $\begin{array}{l}\text { Gold- und Silbermünzen, antike Figuren und } \\
\text { Kunstgegenstände }\end{array}$ & Frage der Herkunft, Echtheit, Forschung \\
\hline Treibstoffe & flüssig & Benzin, Dieselöl & $\begin{array}{l}\text { Toxikologie, Ökologie, Qualitătssicherung. } \\
\text { gesetzliche Verordnung }\end{array}$ \\
\hline Schmierstoffe & flüssig & Motor- und Hydrauliköl, Schmierfett & $\begin{array}{l}\text { Sicherheit, Schadenfälle, Qualitätssicherung, } \\
\text { Tribologie }\end{array}$ \\
\hline Brennstoffe & flüssig, fest & Heizöl, Schweröl, Kohle & Ökologie, Schadenfälle, Qualitătssicherung \\
\hline
\end{tabular}

chemischen Bestimmungsverfahren. Von den heutzutage eingesetzten modernen Instrumentalmethoden werden schwergewichtig spektroskopische Verfahren behandelt, welche in den letzten 20-30 Jahren eine enorme Entwicklung erfahren haben. Der rasche Fortschritt instrumenteller Methoden wurde ermöglicht durch die jüngste Entwicklung der Elektronik und Computertechnik; die Grundlagen der spektroskopischen Techniken waren schon früher bekannt [3]. Nicht atomspektroskopische, d.h. gravimetrische, titrimetrische, elektroanalytische, fotometrische, polarografische und chromatografische Bestimmungsmethoden, sind ergänzend oder alternativ, nach wie vor von Bedeutung oder sogar von wachsender Wichtigkeit. Letzeres gilt insbesondere für chromatografische Verfahren.

Wichtige Einsatz- und Anwendungsgebiete der anorganischen Instrumentalanalytik sind die Forschung, die Mate- rialwissenschaften, die Umweltanalytik inklusive Überwachung (monitoring). Eine qualitativ und quantitativ leistungsfähige Analytik bildet eine wesentliche Voraussetzung zur Erforschung und Entwicklung neuer Werkstoffe und Materialien, der Sicherung von Materialqualitäten, der Erarbeitung aussagekräftigen Datenmaterials zur Beurteilung ökologischer Problemstellungen sowie der Überprüfung der Einhaltung gesetzlicher Vorschriften.

Steigende Anforderungen an die chemische Analytik manifestieren sich durch eine ständig zunehmende Zahl gleichzeitig zu bestimmender Elemente am selben Untersuchungsobjekt. Zusätzlich werden immer tiefere Nachweisgrenzen für die zu messenden Elemente, bzw. Spezies gefordert [4]. Mit nachstehend beschriebenen Verfahren erreichbar sind analytische Nachweise von Elementen im unteren Pikogramm- (pg) und oberen Femtogramm- bereich (fg). Dies entspricht Massenanteilen im Bereich von ppt, je nach Menge an Ausgangsmaterial, wie aus Fig. I zu ersehen ist.

Einen Eindruck von der grossen Vielfalt an Problemstellungen und untersuchten Objekten vermittelt die Zusammenstellung in Tab. 1. Die Matrix, d.h. die chemische Umgebung des oder der betreffenden Elemente oder Spezies ist anorganischer oder auch organischer Natur. Beispiele für letzteren Fall sind Abriebspuren von Metallen in Ölen aus Hydrauliksystemen oder Triebwerken oder Cadmium in Kunststoffen.

Eine analytische Untersuchung beinhaltet grundsätzlich eine Reihe wichtiger Verfahrensschritte, beginnend mit der Problemstellung über die Probenahme bis zur Berichterstattung und Beurteilung (Fig. 2). Jeder einzelne Schritt beeinflusst die Qualität des Resultates und damit die Beurteilung. 
Das Resultat einer analytischen Bestimmung liefert totale Elementanteile unabhängig von der chemischen Bindung, z.B. Stickstoff, Phosphor, Selen oder Cadmium. Es kann auch gezielt bestimmte Spezies betreffen, z.B. Ammonium, Ortho-Phosphat oder Cadmiumselenid. Im Fall der Speziation ist nur derjenige Elementanteil relevant, der im Untersuchungsmaterial in der betreffenden chemischen Form vorliegt.

Der Messbereich der analytischen Elementbestimmung kann ohne weiteres 9 Grössenordnungen und mehr im Massenanteil umfassen (Fig. 1). Diese grosse Spanne ergibt sich, wenn von der Bestimmung von Hauptelementen im Rahmen einer Gesamtanalyse zur Ermittlung der Zusammensetzung eines Untersuchungsmaterials auf die Bestimmung von Elementen im Spuren- oder UItraspurenbereich übergegangen wird.

Instrumentalanalytische Bestimmungsverfahren, wie sie hier behandelt werden, sind durchwegs Relativmessungen. Sie beruhen auf einer eindeutigen Beziehung zwischen dem analytischen Signal und der Zahl der Atome $\mathrm{N}_{0}$ des Analyten in der Probe. Eine grundlegende Voraussetzung für die Qualität des Resultates ist somit in allen Fällen eine sorgfältige Kalibrierung.

Die quantitative Elementbestimmung erfolgt in der Regel an der festen Probe oder an einer Lösung, bzw. der gelösten Probe. Einzelelementbestimmungen werden immer häufiger erweitert auf sogenannte Multielementverfahren, bei denen mehrere Elemente in einem Analysendurchgang gleichzeitig gemessen werden.

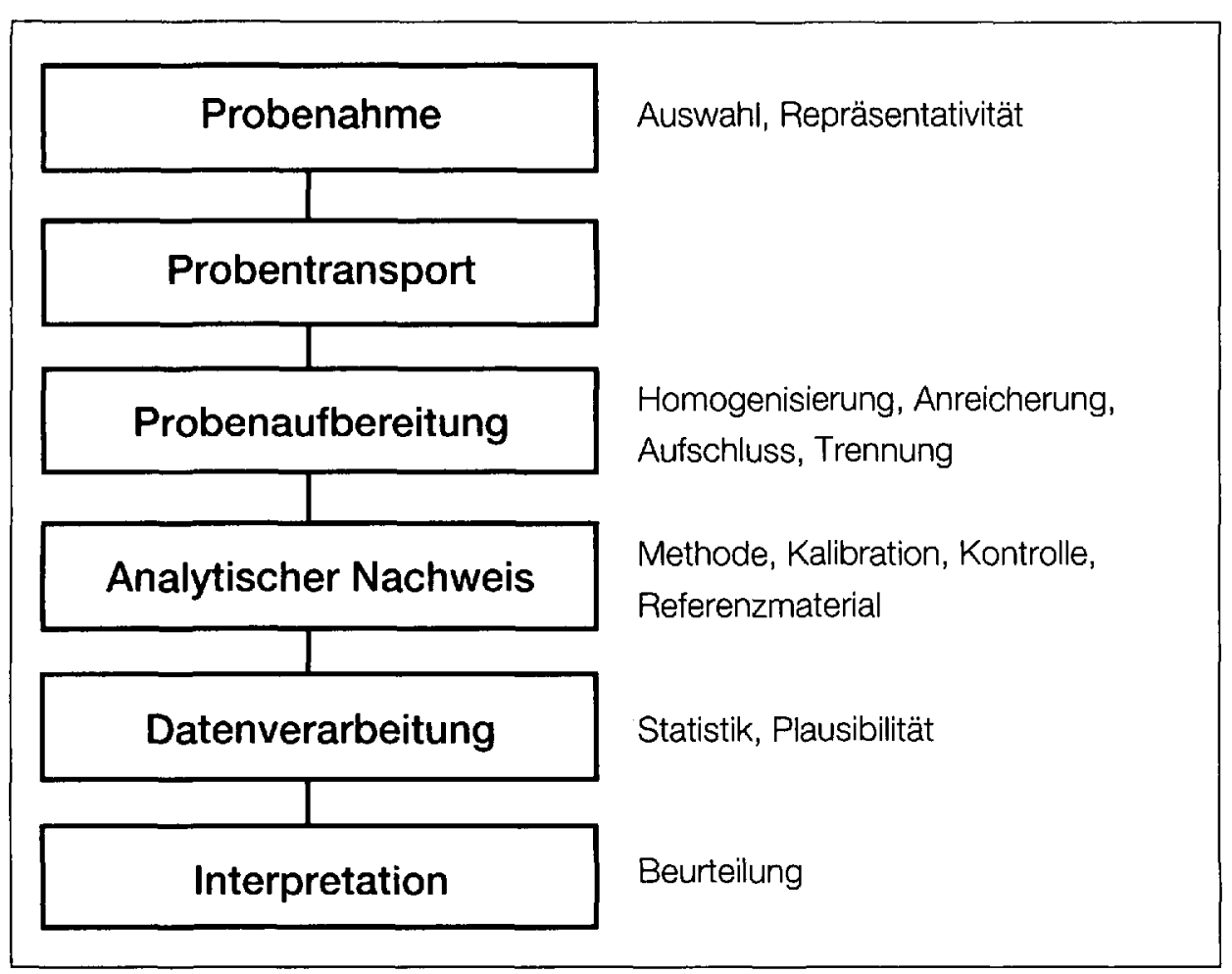

Fig. 2. Verfahrensschritte einer analytischen Untersuchung

Simultanverfahren sind sehr leistungsfähig in Bezug auf Probendurchsatz und Anzahl zu analysierender Elemente pro Zeiteinheit. Beispiele sind Atomemissions-, energiedispersive Röntgenfluoreszenz- und faktisch ICP-Massenspektrometrie. Die grosse Messkapazität, welche sich aus der simultanen Analyse mehrerer Elemente ergibt, kann mit einer Einschränkung der Flexibilität verbunden sein. Dies trifft bei der Atomemissionsspektrometrie (AES) zu, wenn Elemente verlangt sind, die nicht im fest eingebauten Linienprogramm vorgesehen sind. Bei einer Reihe von Verfahren werden mehrere Elemente sequentiell, d.h. nacheinander, jedoch mehr oder weniger im gleichen Analysendurchgang gemessen. Hierzu gehören die sequentielle Emissions- und die wellenlängendispersive Röntgenspektrometrie, bedingt auch die Ionenchromatografie. Die Atomabsorptionsspektrometrie (AAS) ist im allgemeinen rein sequentiell.

Bei den modernen spektrometrischen Methoden mit computergesteuerten Geräten werden für regelmässig anfallende Untersuchungen spezifische analytische Messprogamme entwickelt. Für die eigentliche Messung gestatten die Programme je nach Methode und Stand der Technik halb- oder vollautomatische Abläufe. Das Messprogramm wird mit einer Nachkalibration gestartet, um eine apparative Drift zu korrigieren. Dies erfolgt durch die Messung sogenannter Einstell-, d.h. externer Standardproben, welche die interessierenden Elemente in geeigneter Konzentration enthalten. Die aktuellen appa- mal sein.
iii) Matrix- und Störeinflüsse sollen beherrscht werden. Dazu gehört die simultane Ausgabe aller notwendigen Informationen, z.B. über das gesamte Spektrum.

iv) Die Instrumente müssen einfach zu bedienen sein ('benuitzerfreundlich'), einen hohen Automatisierungsgrad aufweisen sowie adaptiv und selbstdiagnosefähig sein.

v) Die analytische Präzision ist weiter zu verbessern.

\section{Grundlage der Spektrometrie. Anregung und Atomisierung}

Bei den spektrometrischen Bestimmungsverfahren ist die Quantelung der Energie, d.h. die Existenz diskreter energetischer Zustände von Atomen Grundlage der Messung. Für die spektrometrische Analyse muss die Probe angeregt werden, was oft eine vorausgehende Atomisierung bedingt. Atomspektrometrische Instru- 
mente bestehen im wesentlichen aus zwei Hauptkomponenten: dem Atomisierungsund/oder Anregungsteil einerseits und der Messtechnik, d.h. dem Detektionsteil andererseits. In der optischen Atomspektroskopie geschieht die Anregung über die äussersten Elektronen im Atom, im Gegensatz dazu bei der Röntgenspektrometrie über die innersten Elektronen im Atom, worauf später eingegangen wird.

Durch Absorption elektromagnetischer Strahlung bestimmter Wellenlänge erfolgt ein Übergang des Atoms von einem tieferen in einen energetisch höheren Zustand. Beim umgekehrten Vorgang wird elektromagnetische Strahlung emittiert. Die Wellenlänge der betreffenden Strahlung ist umgekehrt proportional der Energiedifferenz der beiden Zustände und charakteristisch für das Element. Es handelt sich um die Spektrallinien der Elemente. Aufgrund diesercharakteristischen Linien lassen sich Elemente qualitativ nachweisen. Die Strahlungsintensität ist proportional der $\mathrm{Ge}$ samtzahl der am Vorgang beteiligten Atome. Dies bildet die Grundlage für eine quantitative Bestimmung. Werden durch die Atomisierung und Anregung auch Ionen in genügender Zahl erzeugt, kann durch massenspektrometrische Detektion, d.h. statt nach der Wellenlänge der Strahlung nach der Atommasse, bzw. dem Verhältnis Masse/Ladung selektioniert werden.

Die Atomisierung und Anregung kann auf verschiedene Arten erfolgen, wie in Fig. 3 schematisch dargestellt ist. Je nach atomphysikalischen Parametern und $\mathrm{Be}$ dingungen im Atomreservoir werden verwendet:

i) die Absorption separat eingestrahlter elektromagnetischer Strahlung zur Detektion und Quantifizierung,

ii) die Emission charakteristischer elektromagnetischer Strahlung und

iii) ionisierte Atome, welche massenspektrometrisch registriert werden.

Atomisierung und Anregung werden im nächsten Abschnitt für jede Methode beschrieben.

\section{Instrumentalanalytische Methoden}

Im folgenden soll kurz auf die wichtigsten Instrumentalmethoden eingegangen werden, wobei es hauptsächlich darum geht, spezifische Vor- und Nachteile im Vergleich zu diskutieren. Wichtige Beurteilungskriterien für die Leistungsfähigkeit einer Instrumentalmethode in der analytischen Praxis sind:

- analysierbare Elemente (und Spezies)

- Probenform (fest, Pulver, flüssig, gasförmig; evtl. elektrische Leitfähigkeit fester Proben)

- Nachweis- und Bestimmungsgrenzen

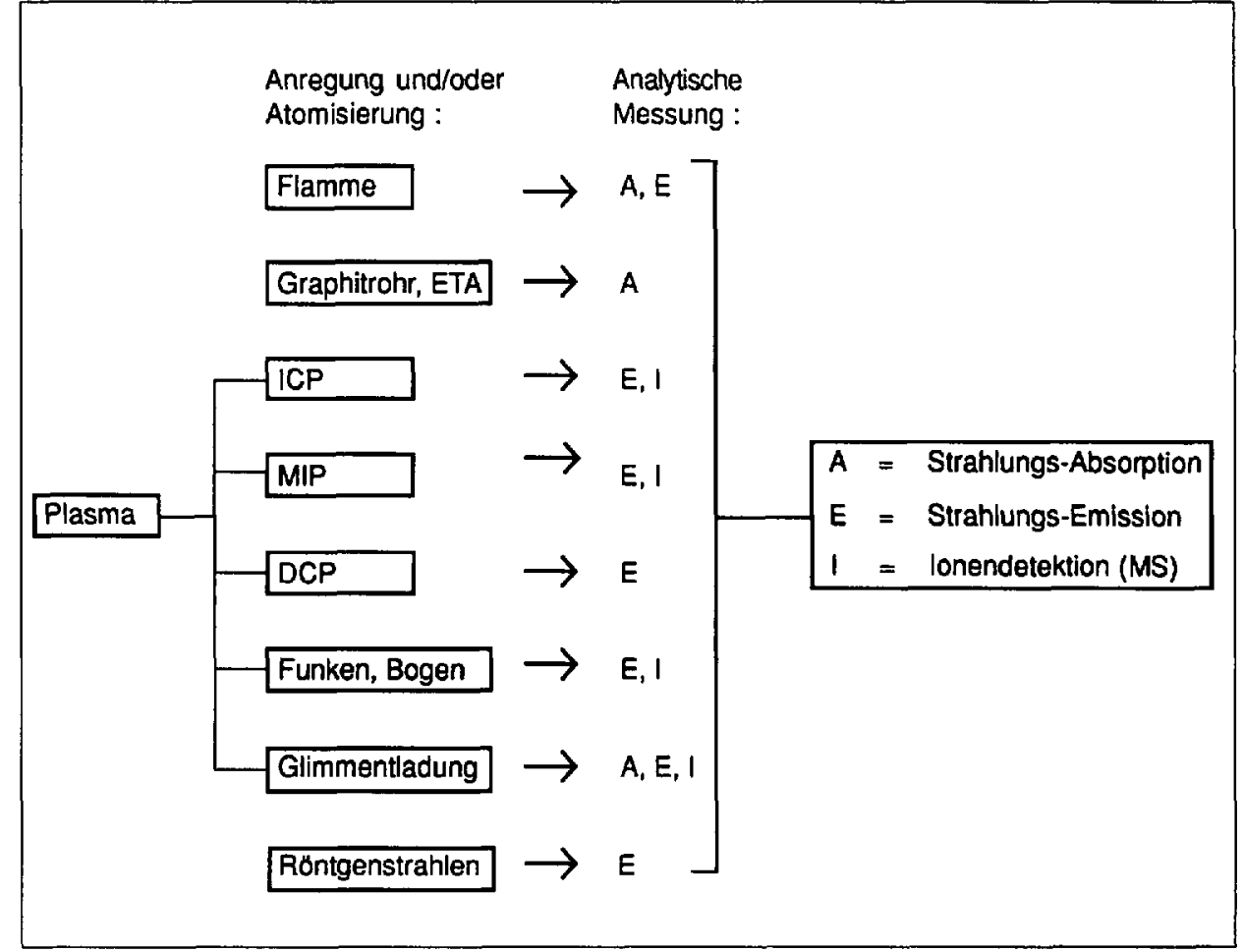

Fig. 3. Anregungs- und Atomisierungsmethoden in der Atomspektrometrie

- Präzision und Genauigkeit

- Messkapazität (Anzahl Proben und Elemente pro Zeiteinheit)

- Messbereich

- Automatisierung

- Eignung für qualitative Übersichtsanalysen

- Kosten, Aufwand

\subsection{Atomabsorptionsspektrometrie}

Die am weitesten verbreitete atomspektrometrische Methode im analytischen Laboratorium ist die Atomabsorptionsspektrometrie (AAS) [5]. Besonders die Flammentechnik (F-AAS) für die quantitative Elementbestimmung in Lösung gehört heutzutage zur Standardausrïstung jedes analytischen Laboratoriums. Bei der Atomabsorption handelt es sich vom Mechanismus her um Elektronenübergänge in der äusseren Atomschale. Dabei werden die in der Flamme durch Verdampfung und Dissoziation erzeugten Atome durch eingestrahlte elektromagnetische Strahlung elementspezifischer Wellenlänge angeregt. Der durch diesen Vorgang im Atomdampf absorbierte Teil der Strahlung wird photoelektrisch gemessen. Für die Kalibration werden gemessene Absorption und Massenkonzentrationen der verwendeten Elementlösungen korreliert. Die resultierende Funktion ist im Idealfall eine Gerade, vielfach jedoch gekrümmt. Aus dieser Funktion kann mittels gemessener Absorption die Massenkonzentration für unbekannte Proben bestimmt werden.

Bei der Technik der flammenlosen Atomabsorption wird die Probe in einem elektrisch beheizten Graphitrohr (GF-
AAS) verdampft. Im Gegensatz zur Flamme erfolgt dadurch eine starke Lokalisierung des Atomdampfes im Rohr. Daraus ergibt sich der Vorteil einer um Grössenordnungen höheren Empfindlichkeit der flammenlosen Technik gegenüber der Flamme. Der Aufheizvorgang im Graphitrohr ist programmierbar. Der Temperaturverlauf kann somit der materialspezifischen Charakteristik der Proben angepasst werden. Neben flüssigen lassen sich auch feste Proben in Mikromengen direkt ins Rohr dosieren. Störende Absorptionen im Atomdampf sind weitgehend kompensierbar, sei dies durch Deuterium- oder Zeernann-Untergrundkompensation.Letztere verwendet die Aufspaltung von Absorptionslinien in einem starken Magnetfeld. Eine spezielle Anwendung der AAS stellt die Kaltdampftechnik für die Quecksilberbestimmung dar. Dabei wird Quecksilber in Lösung zum Metall reduziert und aufgrund seiner Flüchtigkeit als Gas in eine Quarzküvette geleitet, die ihrerseits im Strahlengang eines AAS-Geräts montiert ist. Ähnlich funktioniert die sogenannte Hydridtechnik für Elemente, welche flüchtige Hydride bilden, z.B. Arsen, Selen, Zinn, Antimon. Sowohl bei der Hydridtechnik wie auch bei der Quecksilberbestimmung resultiert durch Abtrennung des Elementes von der Matrix für die Messung ein zusätzlicher Vorteil, weil damit Störungen wegfallen.

Als wichtigste Entwicklungen wurden in den letzten Jahren geräteseitig eine Reihe von Neuerungen eingeführt: automatische Probendosierung ins Graphitrohr, Methoden zur Matrixmodifikation, Untergrundkompensation, Festprobenanalyse, schnel- 
le Aufheizraten für das Graphitrohr. Wesentliche Vor- und Nachteile der Methode sind in Tab. 2 zusammengestellt. Die Zahl jährlicherPublikationen für die Flammentechnik zeigt sinkende Tendenz, die Entwicklung der Methode ist mehr oder weniger abgeschlossen [6]. Hingegen resultiert für die Graphitrohrtechnik immer noch eine Zunahme an Veröffentlichungen, Zeichen für eine recht dynamische methodische Weiterentwicklung. Neue Geräte weisen einen beachtlichen Automatisierungsgrad bezüglich Gerätesteuerung auf.

\subsection{Atomemissionsspektrometrie}

Eine heute stark verbreitete Art der Anregung für flüssige, feste und gasförmige Proben ist die ICP-Methode. Das
Hochtemperaturplasma wird durch induktives Erhitzen von Ar-Gas oder einem anderen Gas erzeugt. Die Analytlösung wird im Ar-Gasstrom zerstäubt und gelangt als Aerosol ins Plasma. Dort wird das Aerosol verdampft, atomisiert, z.T. ionisiert und die Atome und Ionen angeregt. Diese Vorgänge spielen sich während der kurzen Verweilzeit von nur $c a .2$ ms im Plasma ab. Die Plasmatemperatur liegt im Bereich von 5000-10000 . Durch die hohen Temperaturen im Plasma werden bei der ICP-Atomemissionsspektralanalyse (ICP-AES) [7] im Verhältnis zur Atomabsorption eine relativ grössere Anzahl Atome in einen angeregten Zustand überführt. Die angeregten Atome oder Ionen gehen unter Energieabgabe, d.h.

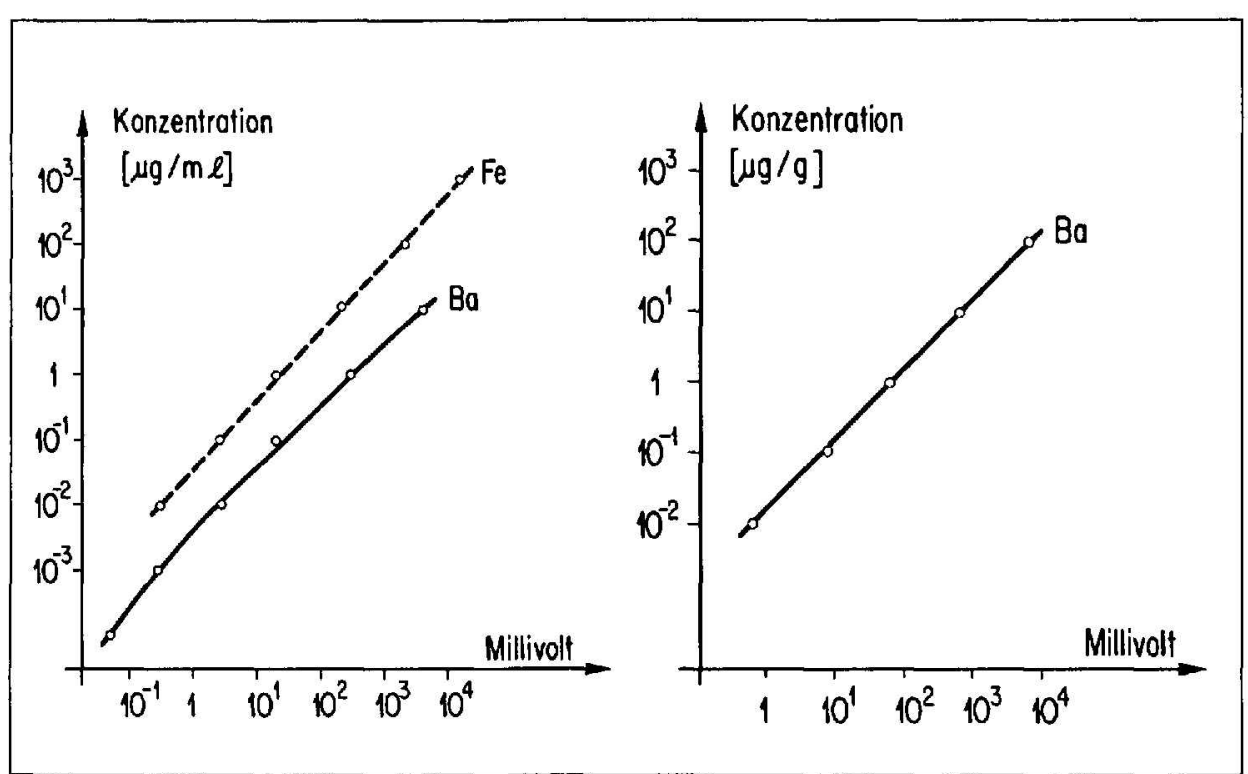

Fig. 4. Linearität des Messignals in Funktion der Konzentration für die ICP-Atomemissionsspektrometrie in w'ässt. (links) und org. Lösung (rechts)

Emission elektromagnetischer Strahlung in den Grundzustand zurück. Das mittels einer Linse gebündelte Licht aus dem Plasma wird auf ein optisches Gitter geleitet, nach Wellenlängen aufgetrennt und die Intensitäten der einzelnen Linien fotoelektrisch gemessen. Die Registrierung der Linien erfolgt mit Fotoröhren, sog. Fotovervielfachern. In einem Simultangerät sind meist zwischen total 20-50 Fotoröhren fest eingebaut, je eine pro Element. Bei den meist verwendeten Spektrometern sind Zahl und Anordnung der Fotoröhren zur Registrierung von Elementlinien vor allem aus geometrischen Gründen beschränkt. Beim Sequenzgerät wird mit einem oder mehreren fahrbaren Monochromatoren, d.h. beispielsweise auf dem Rowlandkreis frei beweglichen Fotoröhren, eine beliebige Wellenlänge angefahren und die Intensität gemessen.

Bei der ICP-AES werden neben Atomlinien mehrheitlich Ionenlinien erzeugt und für die Messung verwendet. Die Intensität einer Spektrallinie ist proportional der Massenkonzentration des betreffenden Elementes im untersuchten Probenmaterial. Bei der AES korrelieren die gemessenen Intensitäten mit den Massenkonzentrationen der Elemente über 4-5 Grössenordnungen meist streng linear. Daraus resultiert ein sehr grosser Messbereich in wässr., aber auch in org. Lösung, wie in Fig. 4 dargestellt. Der grosse lineare Messbereich, die simultane Bestimmung vieler Elemente und die Tatsache, dass bei den hohen Atomisierungstemperaturen chemische Effekte weitgehend eliminiert sind, machen die ICP-AES attraktiv, insbesondere im Vergleich zur Atomabsorp-

Tab. 2. Vergleichende Beurteilung wichtiger Instrumentalmethoden

\begin{tabular}{|c|c|c|c|c|c|c|}
\hline & F-AAS & GF-AAS & ICP-AES & ICP-MS & RFA & IC \\
\hline Verbreitung, Bekanntheit der Methode & + & H & + & + & + & + \\
\hline Zahl analysierbarer Elemente & + & + & ++ & ++ & + & - \\
\hline Probenform, (fest, Pulver, flüssig) & - & + & + & + & + & - \\
\hline Nachweisgrenzen & + & ++ & + & ++ & - & + \\
\hline Präzision & + & - & + & + & ++ & + \\
\hline Messkapazität pro Zeiteinheit & + & + & H & + & H+ & - \\
\hline Dynamischer Messbereich & - & - & + & + & + & - \\
\hline Automatisierung & + & + & + & + & + & + \\
\hline Qualitätive Übersichtsanalyse & - & - & + & ++ & + & + \\
\hline Kosten, Aufwand & + & + & + & -- & - & + \\
\hline $\begin{array}{l}\text { bedeutender Vorteil } \\
\text { mässiger Vorteil } \\
\text { mässiger Nachteil } \\
\text { bedeutender Nachteil }\end{array}$ & & & & & & \\
\hline
\end{tabular}


tion. Mit ICP-AES ist die überwiegende Mehrzahl der Elemente des Periodensystems bestimmbar. Einige der leichten Elemente wie Kohlenstoff, Sauerstoff, Stickstoff, die Halogene sowie Schwefel sind nur beschränkt analysierbar und benötigen ein Vakuumspektrometer, da die betreffenden Emissionslinien im kurzwelligen Spektralbereich liegen und in Luft stark absorbiert werden. Andererseits ist die Methode sehr nachweisstark für andere wichtige leichte Elemente wie Bor, Phosphor und Silicium, welche mit Atomabsorption deutlich weniger nachweisempfindlich gemessen werden können.

Störeinflüsse bei der ICP-AES ergeben sich als Folge der Tatsache der sehr linienreichen Emissionsspektren. Dies führt zu drei hauptsächlichen Interelementeinflüssen:

- direkte Linienüberlappung [8]

- Überlappung durch einen Ausläufer einer benachbarten intensiven Linie

- Erhöhung des Untergrundes durch Streustrahlung, unaufgelöste Molekülbanden.

Der grosse Linienreichtum von Emissionsspektren, beispielsweise im Vergleich zu einem Quadrupol-Massenspektrum oder einem Röntgenspektrum ist aus Fig. 5 ersichtlich. Die Emissionsspektrometrie erfordert aus diesem Grund Spektrometer mit sehr hohem Auflösungsvermögen.

Dennoch auftretende spektrale Störungen, die auch durch gutes Auflösungsvermögen im Spektrometer nicht vermeidbar sind, können quantitativ erfasst und rechnerisch korrigiert werden, was in den betreffenden analytischen Programmen für die Emissionsspektrometrie vorgesehen ist. Genauigkeit und Nachweisvermögen werden dadurch aber eingeschränkt. Zudem muss vorausgesetzt werden, dass der Einfluss des Störelementes bekannt und der Massenanteil dieses Elementes mitbestimmt wird, um die Korrektur quantitativ richtig berücksichtigen zu können.

Simultangeräte sind sehr leistungsfähig bezüglich Messkapazität, jedoch nicht flexibel. Sequenzgeräte sind vielseitig, wobei die Registrierung mehrerer Elemente entsprechend längere Messzeiten und dadurch grössere Mengen an Analysensubstanz bedingt. Simultan-/Sequenzkombigeräte mit einem Anregungsteil und zwei Spektrometern vereinen beide Vorteile. Ein reines Sequenzgerät nutzt im Grunde die typischen Vorteile der Emissionsspektralanalyse relativ schlecht aus.

Für einen optimalen Betrieb von Simultanspektrometern sind bezüglich der Geräteeinstellparameter Kompromisse zu wählen. Die optimalen Messbedingungen sind für die einzelnen Elemente unterschiedlich, insbesondere durch die elementspezifisch verschiedenen Atomisie-

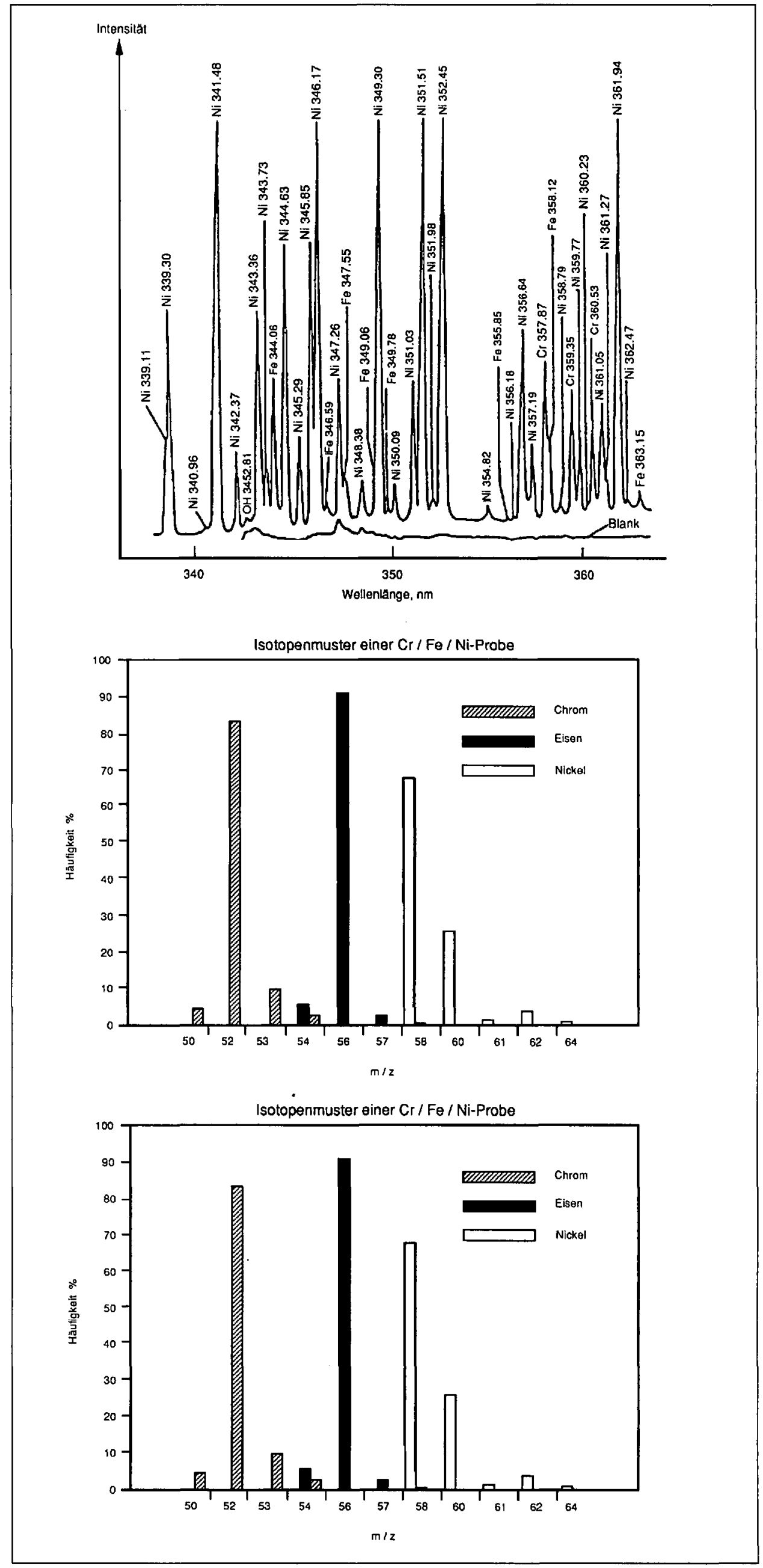

Fig. 5. Unterschiedlicher Linienreichtum eines typischen Emissionsspektrums (oben) im Vergleich at einem ICP-Massenspektrum (Mitte) und einem Röntgenfluoreszenzspektrum (unten) 
rungs- und Ionisierungsbedingungen. Dies äussert sich in den Geräteeinstellparametern Gasstrom, Zerstäuberrate, Beobachtungshöhe im Plasma und Wellenlänge der Spektrallinie.

Ein in vieler Hinsicht Neuland beschreitendes Gerät, welches sehr grosse Messkapazität bei gleichzeitiger Flexibilität offeriert, wurde kürzlich präsentiert. Ein in axialer Richtung beobachtetes Plasma ist mit einem kompakten Echelle-Spektrometer kombiniert. Dieses neuartige Konzept ermöglicht nicht zuletzt eine ausgeprägte Miniaturisierung des Spektrometers [9]. Die spezifischen Vor- und Nachteile der Methode sind in Tab. 2 zusammengefasst.

Beim Ar-Plasma handelt es sich gewissermassen um eine elektrische oder physikalische Flamme, im Gegensatz zu der besser bekannten klassischen, chemischen Flamme, in der ein Redoxvorgang

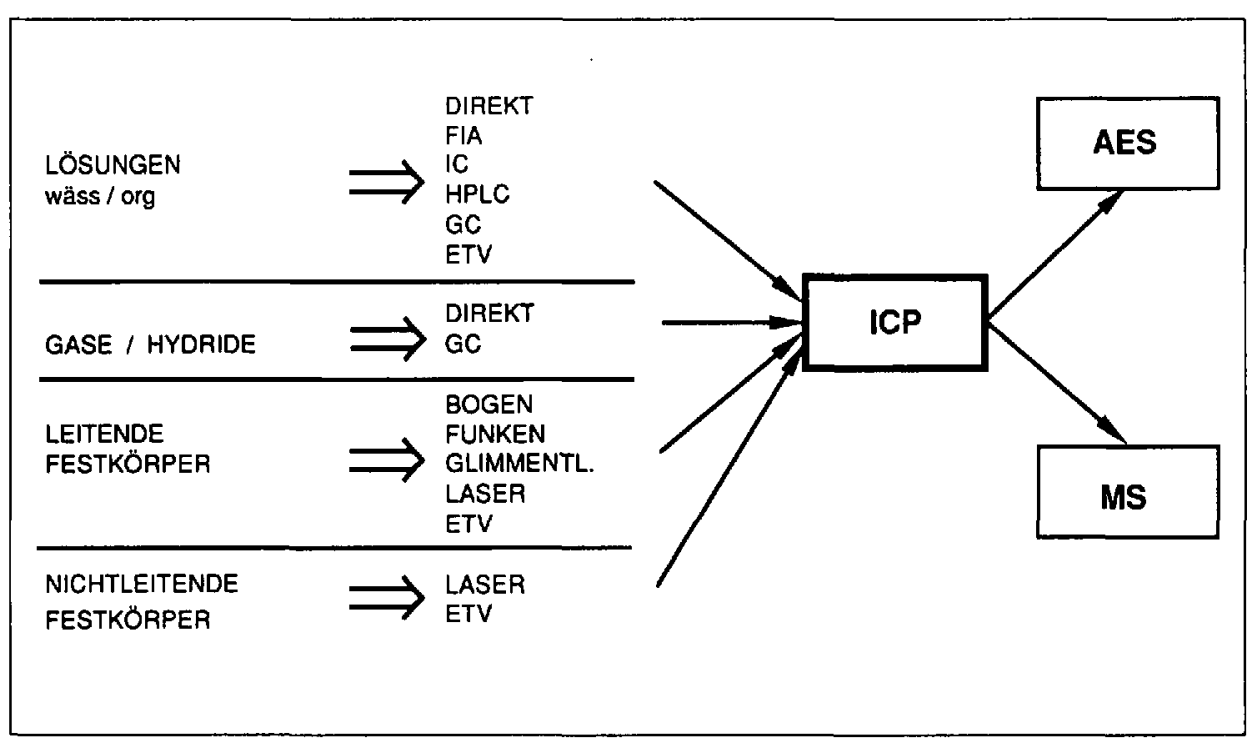

Fig. 6. Probeneinführsysteme im Verbund mit ICP abläuft. Andere Arten von Plasmen werden durch Mikrowellen (MIP) oder im Gleichstrombogen (DCP) erzeugt. Fig. 6 veranschaulicht die vielfältigen Kombinationen von Probenanregungs- und Probeneinführsystemen mit ICP und zeigt eindrücklich die universelle Anwendung des induktiv gekoppelten Ar-Plasmas als Atomquelle in der anorg. Instrumentalanalytik [10].

\subsection{ICP-Massenspektrometrie}

Anorg. Massenspektrometrie mit induktiv gekoppeltem Plasma (ICP-MS) ist eine Analysentechnik zur Bestimmung von Elementen, bzw. Isotopen im Spuren- und Ultraspurenbereich [11]. Das induktiv gekoppelte Plasma, das dem Plasma der ICP-AES entspricht, dient als Ionenquelle. Am Ende der horizontal positionierten Plasmaflamme wird durch eine kleine Öffnung eines Metallkonus (Nickel oder
Platin) ein Teil des Gasstroms, der neben anderen die ionisierten Atome des untersuchten Materials enthält, in das Vakuumsystem des Massenspektrometers abgesaugt. Auf dem Weg vom Gaseintritt zum Detektorteil wird der Gasdruck mittels Turbomolekularpumpen stufenweise vom Normaldruck im ICP auf das erforderliche Hochvakuum von $10^{-6}$ Torr reduziert. Das Quadrupol-Massenspektrometer wirkt als Filter zur Trennung und anschliessenden Detektion der erzeugten Ionen aufgrund ihrer Masse, bzw. des Verhältnisses Masse/Ladung. Fig. 7 zeigt schematisch den Geräteaufbau.

Mit ICP-MS detektierbar sind alle Elemente, welche sich im ICP ionisieren lassen, was für praktisch alle Elemente des Periodensystems zutrifft. Schwache Empfindlichkeiten weisen die Halogene Fluor, Chlor, Brom und Jod auf, welche bevorzugt negative Ionen bilden. Das Quadrupol-Massenspektrometer lässt sich vom üblichen Modus von positiven auf die Detektion negativer Ionen umschalten.

Die Nachweisgrenzen für ICP-MS liegen um einen Faktor 10-100 tiefer als für ICP-AES [12] und damit ungefähr auf dem Niveau der GF-AAS. Für die Elemente mit hohen relativen Atommassen, insbesondere die Lanthaniden und Aktiniden, ist ICP-MS nachweisempfindlicher als die flammenlose Atomabsorption. Gut etabliert hat sich die ICP-MS für die Spurenanalytik in verdünnten wässr. Lösungen, insbesondere für Trink- und Grundwasser.

Ein sehr wichtiger Vorteil des ICPMS-Verfahrens sind die vielfältigen Methoden der Probeneinführung wie Laserablation, elektrothermale Verdampfung im Graphitrohr (ETV), Hydridgenerationsund Fliessinjektionssystem, welche als
Fig. 7. Funktionsschema der ICPMassenspektrometrie

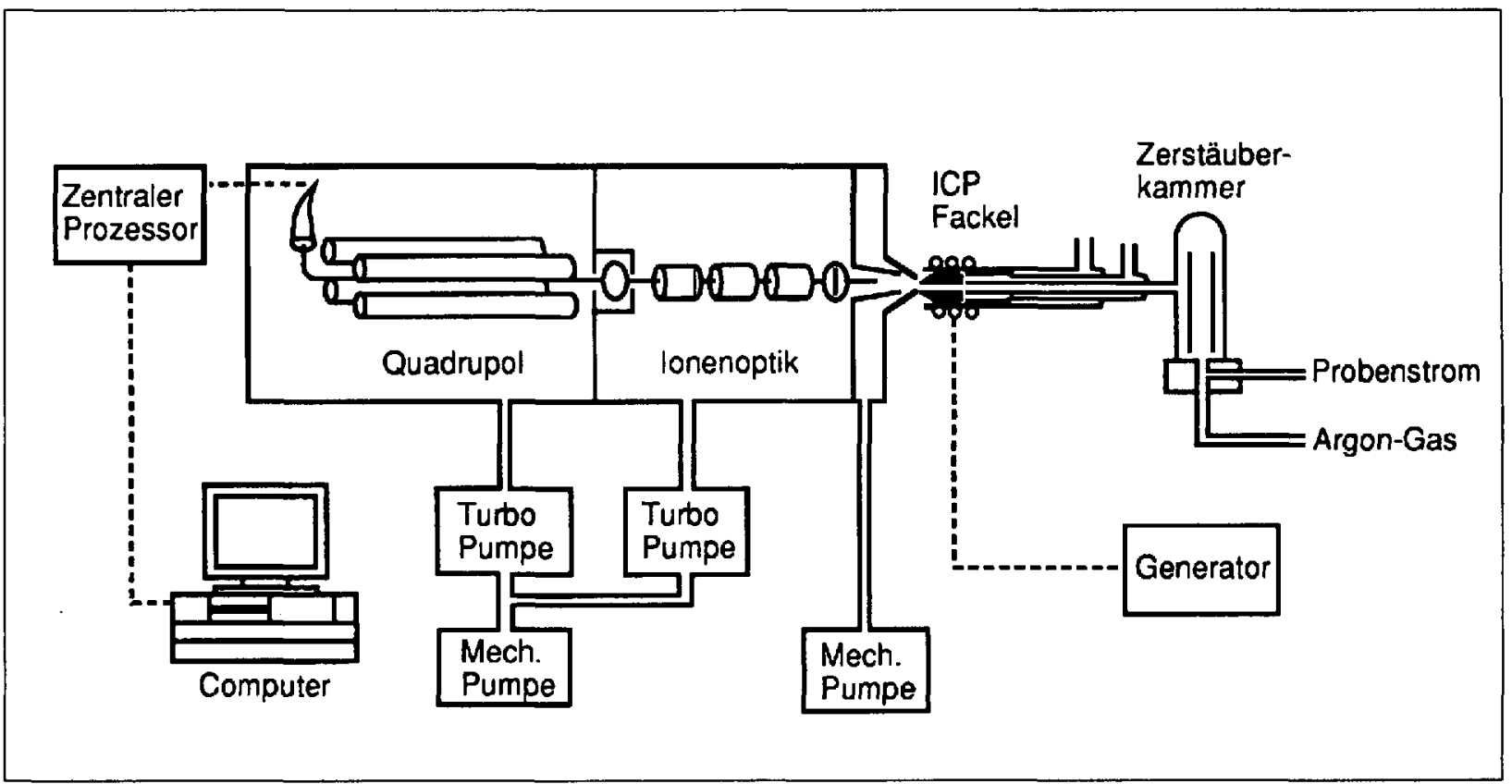


Optionen zum Grundgerät angeboten werden. Die Zuführung flüssiger Proben mittels Fliessinjektion kann das analytische Nachweisvermögen in kritischen Fällen entscheidend verbessern. Mit den verschiedenen Anordnungen können sowohl flüssige wie feste Proben direkt analysiert werden. Für die verschiedenen Aggregatzustände der Probe erfolgt eine Überführung in die Aerosol- oder Gasphase und Einführung ins Plasma via Zerstäubung, Laser-Ablation oder elektrothermale Verdampfung im Graphitrohr, was in Fig. 8 schematisch dargestellt ist. Der zusätzliche Schritt der Überführung einer festen Probe in Lösung kann somit vermieden werden. Für die Festkörperanalyse interessant sind z.B. Bestimmungen von Spuren in hochreinen Werkstoffen im ng/gBereich sowie Spuren an Lanthaniden und anderen seltenen Elementen in mineralischen Stoffen im Massenanteilsbereich ppb bis ppm. Möglich sind auch Untersuchungen von Elementverteilungen an Grenzflächen und die Aufnahme von Tiefenprofilen zum Studium von Grenzschichtphänomenen. Von grossem Nutzen im praktischen Alltag ist mitunter die schnelle, nach weisstarke und rund 70 Elemente des periodischen Systems umfassende halbquantitative Übersichtsanalyse. Die Nachweisstärke der ICP-MS-Methode gestattet detaillierte Bestimmungen an kleinsten Probemengen. Dies ermöglicht eine eigentliche Mikroanalytik auf sehr breiter Elementbasis. Einen zusätzlichen attraktiven Aspekt stellt die Isotopenanalyse dar, welche Aussagen über Zuordnung der Herkunft oder des Ursprungs eines Probenmaterials ermöglichen kann. Die Methode der Isotopenverdünnung gestattet Analysen hoher Präzision auch im Spurenbereich. Dabei werden der Probe künstliche Isotopen als interne Standards zugemischt.

Wie andere Methoden ist auch die ICPMS nicht frei von Störeinflüssen. Es handelt sich um spektrale Interferenzen durch isobare Überlappungen. Insbesondere gehört dazu die Bildung störender, weil signalüberlagernder Molekülionen im Plasma selber oder durch Rekombination während des Durchgangs im Massenspektrometer. Beispiele solcher Überlappungen sind ${ }^{40} \mathrm{Ar}^{16} \mathrm{O}$ mit ${ }^{56} \mathrm{Fe}$ oder ${ }^{40} \mathrm{Ar}^{35} \mathrm{Cl}$ mit ${ }^{75}$ As. Letztere Störung ist insbesondere unerwünscht, da es sich bei Arsen um das einzige Isotop des Elementes handelt und deshalb nicht auf ein anderes ausgewichen werden kann. Als Ausweg neben der nicht immer realisierbaren Vermeidung von Chlor in der Probe bietet sich bei diesem Element die Generierung des Arsens als Hydrid in die Gasphase an, womit eine Trennung von der übrigen Matrix bewerkstelligt wird. Weitere Störmöglich-

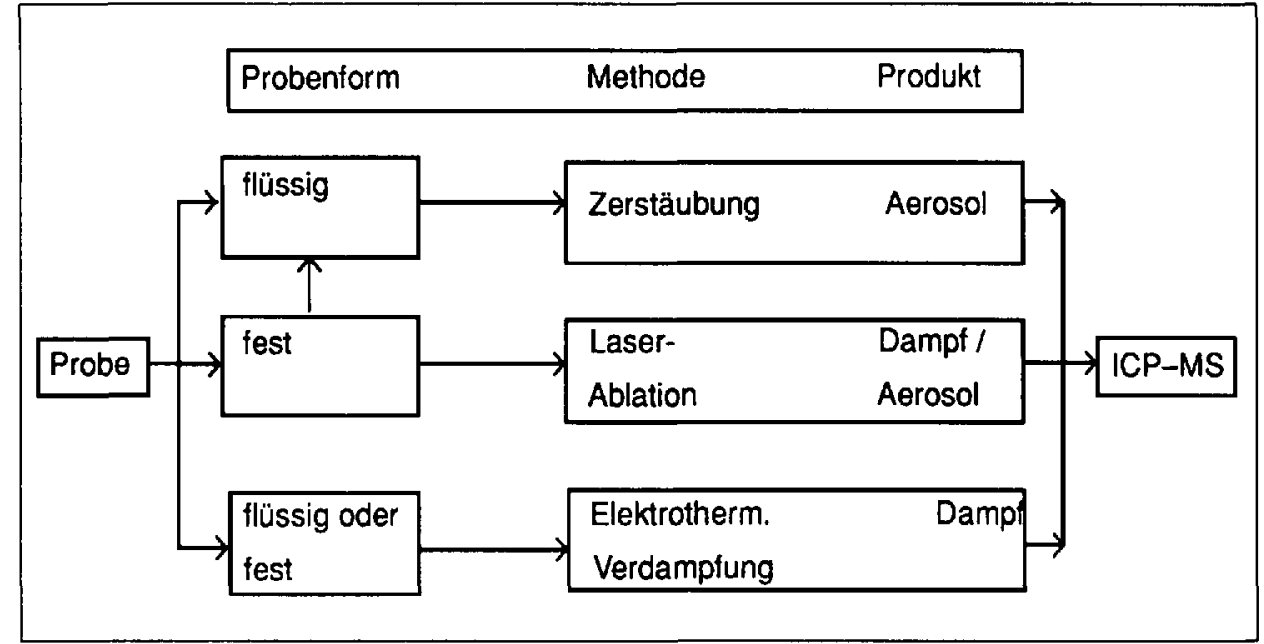

Fig. 8. Schema der Probeneinführung und -anregung für die ICP-Massenspektrometrie

keiten ergeben sich durch Matrixeffekte infolge elektrostatischer Wechselwirkung zwischen den Ionen oder durch Bildung mehrfach geladener Ionen und Oxide.

ICP-MS ist für den Routinebetrieb weitgehend automatisierbar. Angesichts der ausserordentlichen Möglichkeiten und der Vielfalt der Kombinationen von Probeneinführung und Detektion ist ein professioneller Betrieb Voraussetzung, damit das Potential der Methode voll ausgeschöpft und die Investition für die Gerätebeschaffung entsprechend gerechtfertigt sind. Der dazu notwendige personelle und fachliche Aufwand ist nicht zu unterschätzen. Dies gilt ebenfalls für die Infrastruktur. Eine solchermassen nachweisstarke Spurenanalytik stellt hohe Anforderungen an das Umfeld und an die ganze Arbeitstechnik. Dies gilt für die Reinheit der Raumatmosphäre ebenso wie diejenige der Probengefässe und der verwendeten Chemikalien.

ICP-MS hat sich aus der ICP-AES entwickelt und in den letzten Jahren eine enorme Entwicklung erfahren, sowohl bezüglich Technik und Anwendung wie auch Kommerzialisierung. Seit 1984 wurden weltweit über 500 ICP-MS-Geräte installiert, bisher vorwiegend durch zwei Gerätehersteller. Tab. 2 enthält summarisch Vor- und Nachteile der Methode.

Daneben existieren heute bereits Geräte mit einer Kopplung des ICP statt mit dem Quadrupol mit einem hochauflösenden Massenspektrometer für eine selektivere Ionendetektion; diese Geräte sind jedoch sehr kostspielig in der Anschaffung und aufwendig im Betrieb [13].

Eine Variante der massenspektrometrischen Analyse von Festkörpern ist die Glimmentladungs-Massenspektrometrie (GD-MS). Voraussetzung für die Messung ist die elektrische Leitfähigkeit des Probenmaterials. Nichtleitende Materialien werden unter Zumischung von hochreinem Graphit- oder Kupferpulver zu
Presslingen verarbeitet und direkt mit GDMS analysiert.

\subsection{Röntgenfluoreszenz-Spektrometrie}

Die Röntgenfluoreszenzanalyse(RFA) ist ein weiteres wichtiges spektrometrisches Verfahren zur Quantifizierung von Elementanteilen. Das Messprinzip der Röntgenspektrometrie beruht auf der energiereichen Anregung der Atome, meist durch Photonen in Form von Röntgenstrahlen oder durch beschleunigte Elektronen. Die Anregung kann jedoch auch durch Beschuss mit Teilchen aus einem Beschleuniger erfolgen. Durch den Energieeintrag werden in den inneren Elektronenschalen Leerstellen erzeugt, im Gegensatz zur Anregung der äusseren Elektronenschalen bei den vorgängig besprochenen optischen spektroskopischen Methoden im sichtbaren und UV Wellenlängenbereich. Das betreffende Atom gelangt dadurch in einen angeregten Zustand. Augenblicklich, d.h. innerhalb von $10^{-12}$ $10^{-14} \mathrm{~s}$ geht das Atom wieder in einen energieärmeren, bzw. in seinen Grundzustand über. Die Besetzung von Leerstellen erfolgt durch Elektronenuibergänge aus den nächstäusseren Schalen.

Freiwerdende Energie wird teilweise in Form charakteristischer Röntgenstrahlen, der Fluoreszenzstrahlung abgegeben. Aufgrund der zugehörigen Elektronenschalen unterscheidet man zwischen K-, L- und M-Strahlung der Elemente. Die Wellenlänge der Fluoreszenzstrahlung ist charakteristisch für das betreffende Element. Da es sich um Übergänge innerhalb der innersten Schalen handelt, sind die Energien weitgehend unabhängig von der Bindungsform des Atoms. Lediglich bei leichten Atomen ist ein Einfluss chemischer Bindungen auf die Energie und damit die Wellenlänge der Fluoreszenzstrahlung feststellbar. Die Intensität einer Elementlinie ist ein direktes Mass für die Konzentration des Elementes im unter- 
suchten Material. Die Methode arbeitet zerstörungsfrei; eine Atomisierung ist nicht notwendig.

Der Zusammenhang zwischen gemessener Intensität und Massenanteil ist stark abhängig von der Matrix, d.h. der Zusammensetzung des untersuchten Probenmaterials bezüglich weiterer Elemente. Der Einfluss der übrigen Elemente auf die Fluoreszenzintensität kann sich sowohl abschwächend auswirken infolge Massenabsorption wie auch verstärkend infolge Sekundäranregung. Die Austrittstiefe der Fluoreszenzstrahlung liegt in der Grössenordnung von $100 \mu \mathrm{m}$ für eine Festprobe. Sie ist abhängig von der Ordnungszahl der im Material vorhandenen Atome und der Wellenlänge der gemessenen Strahlung.

Die Nachweisgrenze liegt für die meisten Elemente im unteren ppm-Bereich. Beim Übergang zu leichten Elementen, etwa ab Natrium im Periodensystem, wird das Nachweisvermögen rasch schlechter. Einflüsse von Matrix und Korngrösse auf die Intensität der Fluoreszenzstrahlung sind für die Quantifizierung zu berücksichtigen. Es sind heute leistungsfähige mathematische Modelle für die Korrektur von Matrixeffekten verfügbar. Mittels Algorithmen sind effektive Elementanteile aus den experimentellen Messwerten unter Berücksichtigung der Einflussparameter berechenbar. Die Röntgenfluoreszenzspektren sind recht linienarm, d.h. elementspezifisch [14].

Die Standardmesseinrichtung für die wellenlängendispersive Röntgenfluores- zenzanalyse besteht aus einer Röntgenröhre zur Erzeugung der Strahlung für die Probenanregung sowie einem Analysatorkristall und einem Goniometer mit einem Detektionssystem und Datenverarbeitung zur Registrierung und Auswertung der emittierten charakteristischen Fluoreszenzstrahlung. Sobald das Gerät entsprechend kalibriert ist, kann es vollautomatisch betrieben werden. Für flüssige Probenmaterialien kommen neben der $\mathrm{Di}$ rektbestimmung auch Anreicherungsverfahren zur Anwendung, was einen gewissen experimentellen Aufwand bedingt, jedoch zu besseren Nachweisgrenzen führt.

Es ist bemerkenswert, dass bis 1987 weltweit 14000 wellenlängendispersive Geräte installiert worden sind. Wichtige Anwendungen neben dem Einsatz im analytischen Laboratorium sind industrielle Qualitätsuiberwachungen in der Produktion, z.B. bei metallverarbeitenden Betrieben, Zementwerken oder in der pharmazeutischen Industrie. Typische Vorzüge und Nachteile der Methode gehen aus Tab. 2 hervor.

Eine alternative Entwicklung stellt die energiedispersive Röntgenfluoreszenz dar, welche mit hochauflösenden Halbleiterdetektoren arbeitet. Der apparative Aufwand und damit die Gerätekosten sind geringer als bei der wellenlängendispersiven Technik, jedoch auch die analytische Leistungsfähigkeit. Für Spurenbestimmungen hat in den letzten Jahren die Röntgenfluoreszenz mittels Totalreflexion eine gewisse Bedeutung erlangt [15].

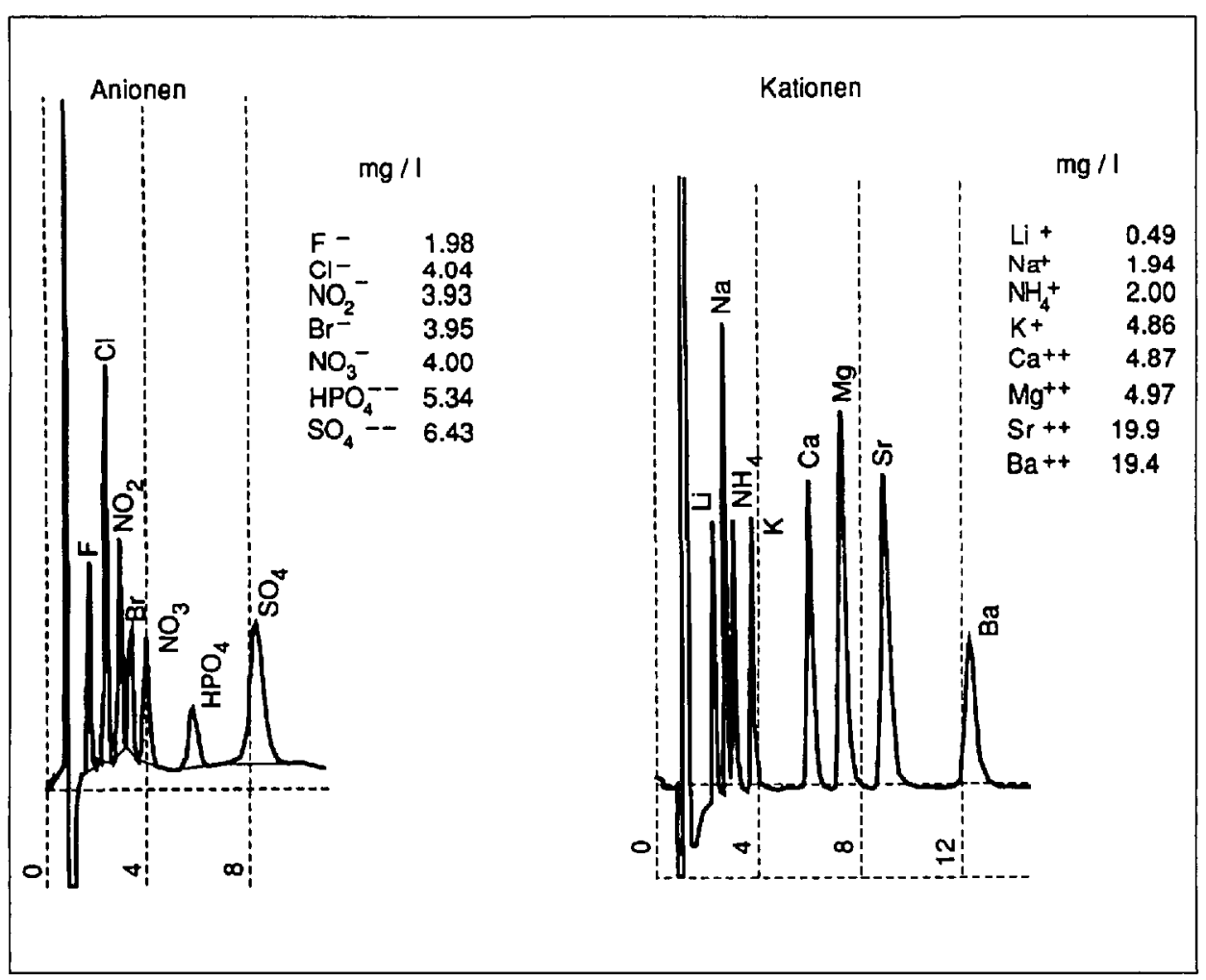

Fig. 9. Ionenchromatogramme für Anionen (links) und Kationen (rechts)

\subsection{Ionenchromatografie}

Es handelt sich um eine nicht-atomspektrometrische Methode zur Bestimmung anorg. und org. Ionen in wässr. Medien. Die Ionenchromatografie (IC) dient der quantitativen Bestimmung von Spezies durch chemische Auftrennung und anschliessender Detektion und ist recht nachweisstark. Sie gestattet die Ausführung qualitativer Übersichtsanalysen an Proben unbekannter Zusammensetzung. Das Verfahren hat in den letzten Jahren die Anionenanalytik entscheidend verbessert. In Fig. 9 sind zwei Chromatogramme für typische Anionen und Kationen wiedergegeben. Gerade in der Anionenanalytik ist die Speziation ein wichtiges Argument für die IC. Chemische Störeinflüsse durch in der Lösung vorliegende Fremdkomponenten müssen allerdings berücksichtigt werden.

\subsection{Weitere Methoden}

In der anorg. Analytik stehen eine ganze Reihe weiterer instrumenteller Methoden im Einsatz. Neben elektroanalytischen Verfahren, Verbrennungsanalysen vor allem für die Bestimmung leichter Elemente wie Kohlenstoff, Stickstoff, Schwefel, sind es verschiedene chromatografische Verfahren. Verbundsysteme, in denen verschiedene Methoden gekoppelt werden, sind von steigender Bedeutung. Beispiel ist die Kombination von Gaschromatografie mit Atomemission als Detektionssystem.

Die Polarografie ist eine seit längerer Zeitetablierte, nachweisstarke analytische Methode für Lösungen. Der instrumentelle Aufwand ist relativ bescheiden. Das Verfahren eignet sich für definierte Anwendungsbereiche und bestimmte Elemente. Die Fliessinjektionsanalyse (FIA) wurde bereits erwähnt. Sie funktioniert als Probeneinführungssystem oder direkt zur Bestimmung in Lösung mittels chemischer Umsetzung oder Detektion eines Signals. FIA lässt sich mit den verschiedensten Techniken kombinieren [16]. Sie ist insbesondere in jenen Fällen der Lösungsanalyse sehr vorteilhaft, in denen entweder nur sehr kleine Volumina zur Verfügung stehen oder hohe Konzentrationen an Fremdionen stören.

Neben der Neutronenaktivierungsanalyse als leistungsfähiger, jedoch aufwendiger Methode in der Spurenanalytik ist die Elektronenstrahlmikrosondenanalyse erwähnenswert. Sie hat Bedeutung erlangt für Lokalanalysen an Festproben. Die Elementbestimmung erfolgt qualitativ und quantitativ aufgrund der Analyse sekundär erzeugter Röntgenstrahlen. Für Spurenbestimmungen setzt allerdings die Nachweisempfindlichkeit im allgemeinen bald Grenzen. 
Ein Bündel von Methoden steht für die Charakterisierung von Oberflächen bezüglich der obersten Atomlagen sowie von dünnsten Grenzschichten mit Elektronenspektroskopie zur chemischen Analyse(ESCA), Augerelektronenspektroskopie (AUGER, AES) und Sekundär-IonenMassen-Spektrometrie (SIMS) zur Verfuigung [17].

Neue sich in Entwicklung befindende, äusserst nachweisstarke analytische Techniken beruhen auf Laser-Spektroskopie. Beispiele sind die Resonanzionenspektroskopie (RIS) und die davon abgeleitete Resonanz-Ionisations-Massenspektrometrie (RIMS) [18]. Es würde den Rahmen dieser Arbeit sprengen, näher auf diese Verfahren einzutreten.

\section{Methodenvergleich}

Ein Methodenvergleich muss angesichts der grossen Vielfalt und Möglichkeiten, aber auch der sehr unterschiedlichen Anforderung analytischer Laboratorien recht schematisch bleiben und soll sich in Ergänzung des Vergleichs in Tab. 2 auf eine direkte Gegenüberstellung von vier Kriterien beschränken:

- Nachweisgrenzen

- Messbereich

- erreichbare Präzision

- Gerätekosten

\subsection{Nachweisgrenzen}

Zwei Bemerkungen zu den Nachweisgrenzen (detection limit):

i) die Nachweisgrenze ist ein statistischer Wert und definiert als diejenige Massenkonzentration eines Elementes, welche eine Signalintensität liefert, die z.B. je nach Konvention dem Dreifachen der Standardabweichung des Untergrundsignals entspricht:

$c_{\mathrm{dl}}=3^{*} \mathrm{~s}_{\mathrm{u}}$

$c_{\mathrm{dl}}=$ Nachweisgrenze (Massenkonzentration, z.B. $\mu \mathrm{g} / \mathrm{ml}$ )

$s_{u}=$ Standardabweichung vom Mittelwert der Untergrundmessung (z.B. aus zehn wiederholten Messungen)

ii) $c_{\mathrm{dl}}$ ist als instrumentelle Nachweisgrenze definiert. Die im praktischen Routinebetrieb erreichbaren Nachweisgrenzen sind um einen Faktor zwei bis fünf schlechter, z.B. durch Addition von Blindwerten und anderen Einflüssen. Man spricht dabei von den für die Praxis viel wichtigeren Bestimmungsgrenzen.

Typische Nachweisgrenzen für die besprochenen Instrumentalmethoden sind aus Fig. 10 ersichtlich. Sie sind als Konzentrationsbereiche dargestellt. Die Angabe als Bereiche ist insofern sinnvoll, als die Nachweisgrenzen für verschiedene

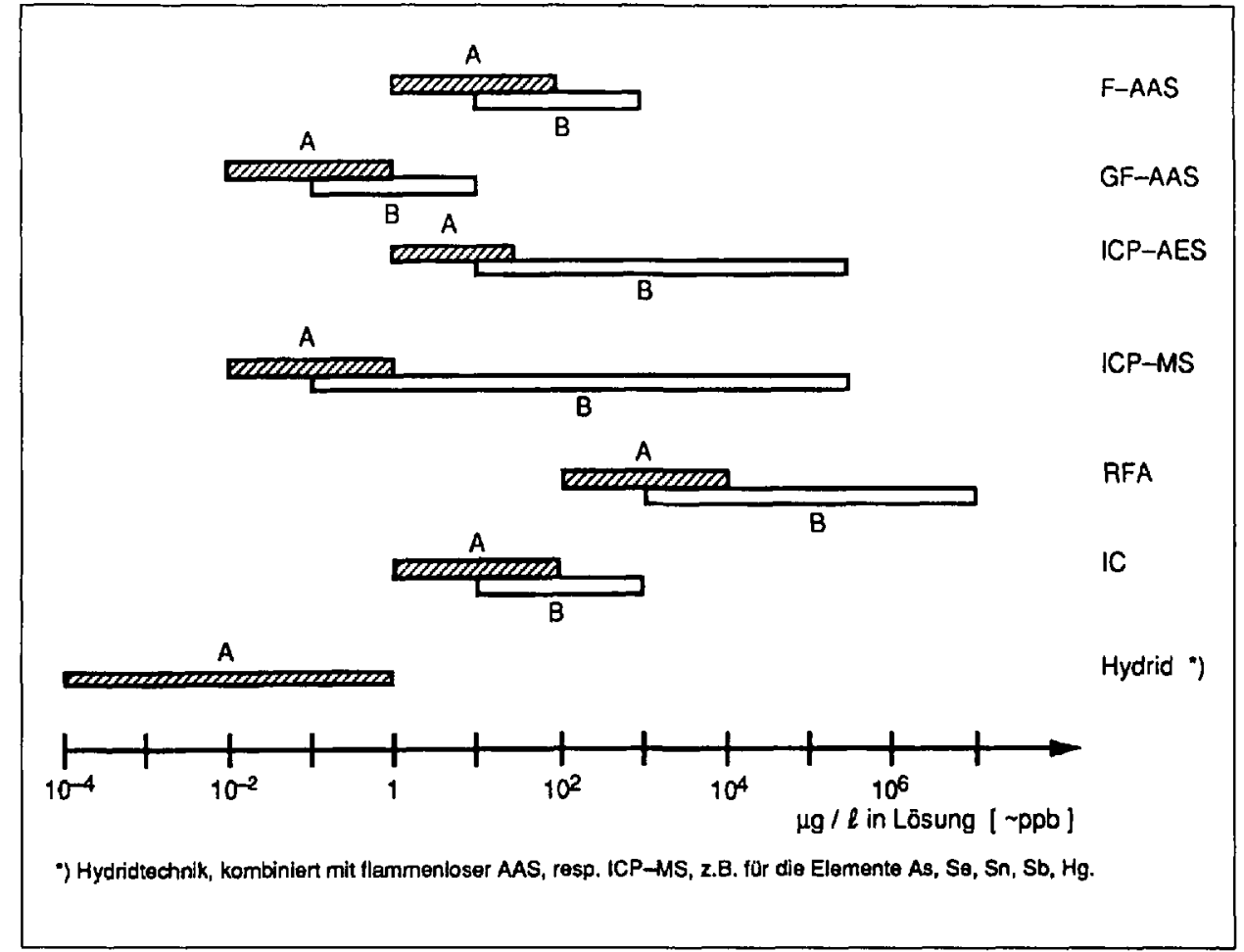

Fig. 10. Nachweisgrenzen (A) und Messbereich (B) verschiedener Instrumentalmethoden (Nachweisgrenzen in Lösung. Auf die Festprobe bezogen liegen die Nachweisgrenzen generell um einen Faktor 100 höher).

Elemente bei jeder Methode variieren, für gewisse Methoden sogar stark variieren können [19]. Unter Nachweisvermögen (detection power) wird die kleinste Massenkonzentration einer Substanz verstanden, die mit der Methode detektiert werden kann

\subsection{Messbereich}

Fig. 10 stellt ebenfalls typische Arbeitsbereiche für die Methoden FlammenAtomabsorption, Graphitrohr-Atomabsorption, ICP-Atomemissions-, ICP-Massenspektrometrie und Röntgenfluoreszenzspektrometrie dar. Unter dem Begriff Messbereich ('dynamischer Messbereich') wird das Konzentrationsintervall eines Elementes verstanden, innerhalb dessen eine instrumentelle Bestimmung ohne zusätzliche Verdünnung und ohne Qualitätseinbusse im Resultat erfolgen kann. Unter diesem Gesichtspunkt sind beide ICPspektrometrischen Methoden, ICP-AES und ICP-MS, der Atomabsorption klar überlegen, indem über einen sehr grossen Konzentrationsbereich mit einer einzigen Kalibration gemessen werden kann.

\subsection{Präzision und Genauigkeit}

- Präzision (precision) bzw. Reproduzierbarkeit, bezieht sich auf "interne Kalibration', d.h. Messungen im gleichen Laboratorium. Sie wird durch Bestimmung mit einer Methode an verschiedenen Probenserien über einen längeren Zeitraum ermittelt.

- Genauigkeit (accuracy) bzw. Richtig- keit bezieht sich auf 'externe Kalibration' oder externe Kontrolle mit gut charakterisierten externen Proben oder Standard-Referenzmaterialien, welche durch viele verschiedene analytische Laboratorien nach mehreren zuverlässigen und unabhängigen Methoden analysiert worden sind [20]. Die Genauigkeit ist normalerweise schlechter als die Präzision. Der Begriff Genauigkeit wird benutzt, um die gesamten Abweichungen, systematische wie zufällige, zu beschreiben. In die Genauigkeit gehen sowohl die Probenvorbereitung (Einwaage, Löseoperation, Verdünnungsfaktor, Abweichungen durch Kontamination oder Verlust) als auch die eigentliche Messung (Störeinflüsse durch die Matrix bzw. Drittelemente) ein. Im ungünstigsten Fall summieren sich die einzelnen Abweichungen. Die Genauigkeit ist viel schwieriger zu quantifizieren als die Präzision.

Die optimal erzielbare Präzision der ICP-AES-Messung beispielsweise liegt bei sorgfältiger Ausführung bei rund $1 \%$ relativ, in der Praxis zwischen ein bis mehreren Prozent [21]. Dies bedeutet konkret, dass eine wiederholte Messung an der gleichen Lösung an verschiedenen Tagen, jeweils mit einer Nachkalibration einen Mittelwert mit einer Standardabweichung in dieser Grössenordnung liefert. Voraussetzung ist eine Messung in einem Massenkonzentrationsbereich, der mindestens dem Hundertfachen der Nachweisgrenze 
entspricht. Bei Konzentrationen näher der Nachweisgrenze wird die Präzision aus statistischen Gründen schlechter. Die apparativ erreichbare Präzision resultiert aus der Gerätestabilität, der Stabilität der Zerstäubung und des Plasmas. Eine gute Präzision ist Voraussetzung, jedoch nicht Garantie für ein richtiges Analysenergebnis, d.h. für eine gute Genauigkeit.

Die erreichbaren Präzisionen für die verschiedenen hier beschriebenen instrumentellen Methoden liegen analog der ICP-AES im günstigsten Fall bei ca. $1 \%$ relativ, wobei die Einhaltung dieser Präzision im Routinebetrieb, wie erwähnt, hohe Anforderungen an die Methode und deren Betreiber stellt. Bessere Präzision bis $c a$. $0,3 \%$ relativ ist mit Röntgenfluoreszenz erreichbar, womit die RFA gleichzeitig zur genauesten der beschriebenen Methode wird. Sie wird höchstens noch mit aufwendigeren Isotopenverdünnungsanalysen und mit nass-chemischen Verfahren erreicht. Etwas schlechtere Präzisionen liefert generell die Graphitrohr-Atomabsorption.

Tab. 3. Typische Beschaffungskosten für Geräte der anorg. Instrumentalanalytik

\begin{tabular}{ll} 
& kFr. $\left.{ }^{2}\right)$ \\
F-AAS & $20-100$ \\
GF-AAS & $100-200$ \\
ICP-AES & $200-300$ \\
ICP-MS & $500-900$ \\
RFA & $200-400$ \\
IC & $30-100$ \\
\hline
\end{tabular}

a) $\mathrm{kFr} .=\mathrm{SFr}$. 1000.-

Tab. 4. Methoden der Probenvorbehandlung für die Instrumentalanalytik

$\begin{array}{ll}\text { flüssig/flüssig: } & \text { Verdünnung } \\ & \text { Anreicherung } \\ & \text { Addition } \\ & \text { Extraktion } \\ & \text { Chromatografie } \\ \text { fest } \rightarrow \text { flüssig: } & \text { Lösen } \\ & \text { Nassaufschluss (Temperatur, Druck, } \\ & \text { Mikrowellen, Chemie) } \\ & \text { Verbrennung, Veraschung/Lösen } \\ \text { fest/fest: } & \text { Schmelzen/Lösen } \\ & \text { Mahlen, Homogenisieren } \\ & \text { Schmelzen, Vergiessen } \\ & \text { Verbrennen, Veraschen } \\ & \text { Schleifen, Polieren } \\ & \text { Pressen } \\ \text { flüssig } \rightarrow \text { fest: } & \text { Oberflächenbeschichtung } \\ & \text { Fallung } \\ & \text { Adsorption } \\ & \text { Extraktion } \\ & \text { Gefriertrocknung }\end{array}$

Damit sich diese erreichbaren Präzisionen in entsprechend guten Genauigkeiten niederschlagen, ist eine sehr sorgfältige Geräte-Kalibrierung vorauszusetzen. Eine Ein- oder Zweipunktkalibration mag für eine rasche qualitative Bestimmung genügen. Quantitativen Bestimmungen sind Mehrpunktkalibrationen mit möglichst homogen über den gesamten Messbereich verteilten Werten zugrunde zulegen. Die Funktion wird rechnerisch mit einem Polynom möglichst niedrigen Grades beschrieben, eine Fünfpunktkalibration für ICP-AES beispielsweise möglichst miteinem Polynom zweiten, maximal dritten Grades. Die Verwendung von Polynomen höherer Ordnung mag zwar scheinbar bessere Übereinstimmung mit den experimentellen Werten der Kalibrierung ergeben, täuscht jedoch eine bessere Qualität vor als effektiv vorhanden ist und führt zu systematischen Fehlern.

Bei der Ermittlung der Zusammensetzung eines metallischen Werkstoffes beispielsweise sind bei Gehalten im Prozentbereich Genauigkeiten von $0,5-3 \%$ relativ je nach Element und Gehalt zur sicheren Zuordnung zu einer Werkstoff-Nummer unabdingbar. Hier stösst man mit verschiedenen spektrometrischen Verfahren bezüglich Präzision bereits an die Grenzen der Möglichkeiten. Mit konventionellen nass-chemischen Analysen wie Titrimetrie, Gravimetrie oder Coulometrie sind hingegen im Element-Massenbereich 1$100 \%$ derartige Relativgenauigkeiten durchaus realisierbar [22].

\subsection{Beschaffungskosten}

Tab. 3 gibt einen Anhaltspunkt für typische Beschaffungskosten für Geräte der hier besprochenen Instrumentalanaly- semethoden. Ein Zusammenhang zwischen Gerätekosten und analytischer Leistungsfähigkeit ist unübersehbar. Teurere Geräte haben im Markt nur eine Chance, wenn damit entweder kapazitätsmässig hohe Probendurchsätze mit guten Resultaten erzielt oder aber sehr anspruchsvolle analytische Problemstellungen gelöst werden können.

\subsection{Zusammenfassende Beurteilung}

Obwohl sich viele analytische Bestimmungen in der Praxis grundsätzlich mit mehr als einer der behandelten instrumentellen Verfahren ausführen lassen, steht gewöhnlich eine Methode im Vordergrund. Kriterien für die Wahl des Verfahrens sind Art der Fragestellung, Probenmaterial, form und -zahl, Art und Zahl zu bestimmender Elemente, Konzentrationsbereich, erforderliche Genauigkeit und Nachweisvermögen sowie die instrumentelle Dotierung des analytischen Laboratoriums. Randbedingungen wie Menge an verfügbarem Probenmaterial, eine eventuelle Forderung nach zerstörungsfreier Prüfung - z.B. bei antiken Schmuckstücken oder Münzen - der erforderliche Arbeitsaufwand und die Terminfrage sind oft methodenentscheidend. Vom analytischen $\mathrm{Ge}-$ sichtspunkt ist die Möglichkeit der Überprüfung einer Bestimmung mittels einer zweiten unabhängigen Methode ausserordentlich nützlich und wünschbar, um systematische Fehler auszuschliessen. Darin liegt nicht zuletzt der Vorteil eines breitgefächerten Instrumentariums.

Der Trend nach tieferen Nachweisgrenzen und einer ständig steigenden Zahl gleichzeitig zu bestimmender Elemente ist nicht ohne Einfluss auf Aufwand und Kosten. Je nachweisstärker ein Verfahren, umso höher der Aufwand und die Kosten. Nachweisvermögen, Zuverlässigkeit und Wirtschaftlichkeit sind komplex miteinander vernetzt und verlangen eine Optimierung je nach Aufgabenstellung.

\section{Probenahme, Probenvorbehandlung}

Die Probenahme ist ein wichtiger, oft entscheidender Schritt der Analyse. Die aufwendigste und sorgfältigste analytische Messung ist wertlos, wenn die Probenahme nicht repräsentativ, nicht vollständig oder sonst fehlerhaft erfolgt. Ziel einer sorgfältigen Probenahme ist die Repräsentativität des zu untersuchenden Labormusters bzw. der Muster im Hinblick auf die gesamte Charge und die Fragestellung. Von vergleichbarer Wichtigkeit wie die Probenahme ist die Probenvorbehandlung; wichtige Operationen sind in Tab. 4 aufgelistet. Die Probenvorbereitung muss bezüglich der analysierten Komponenten 


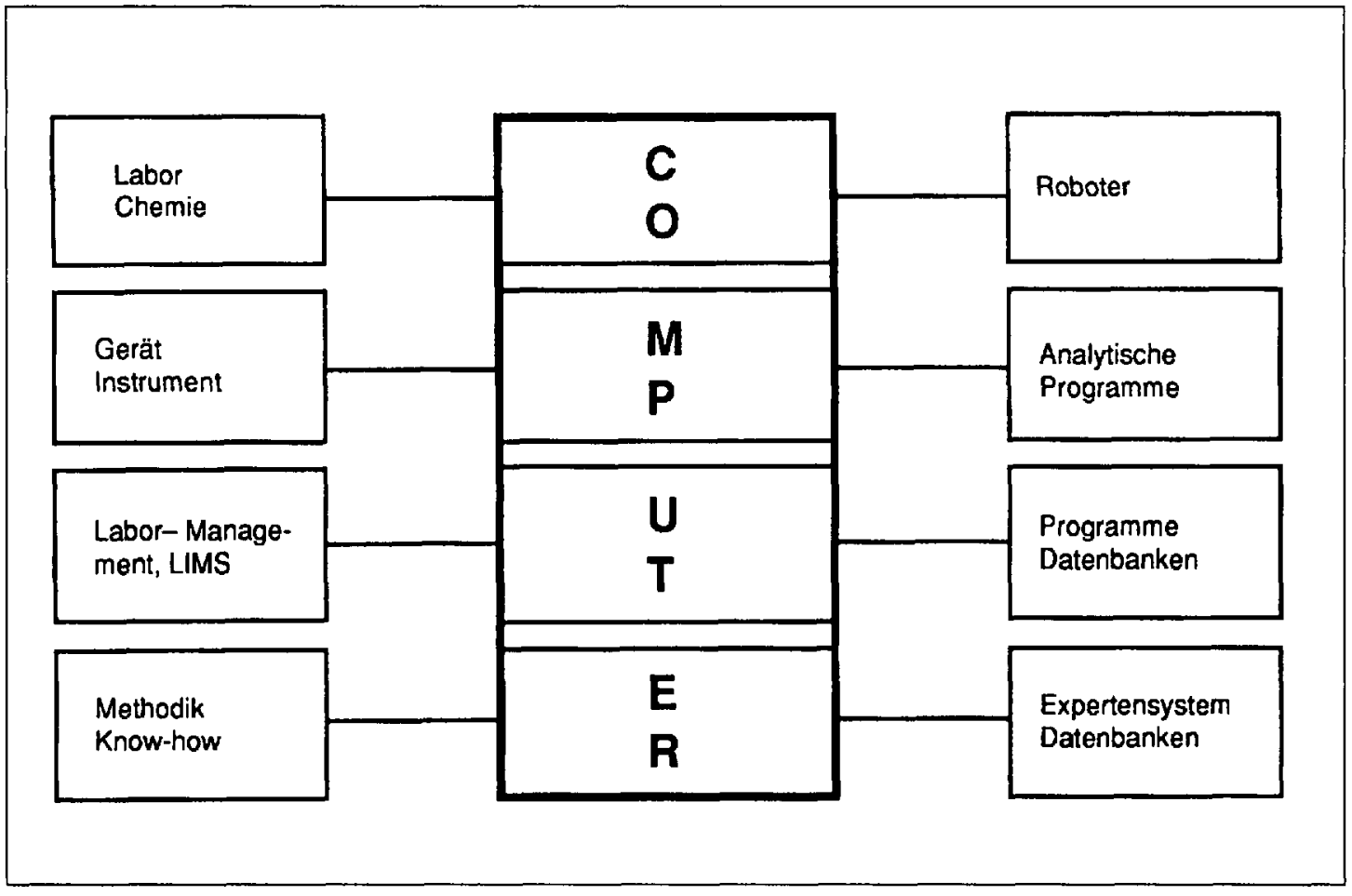

Fig. 11. Vier Ebenen der LahorAutomatisierung verlust- und kontaminationsfrei erfolgen. Unter diesem Gesichtspunkt gilt insbesondere für die Spurenanalytik der Grundsatz, dass ein Direktbestimmungsverfahren gegenüber einem Prozedere, das mehrere Behandlungsschritte umfasst, vorzuziehen ist. Jeder zusätzliche operationelle Schritt ist eine potentielle Quelle für Kontamination oder Verlust.

In der anorg. Analytik werden häufig feste Proben für die Elementanalyse in Lösung überführt. Der Grund liegt in der Verfügbarkeit leistungsfähiger und nachweisstarker Verfahren zur Elementbestimmung in Lösung, wie Atomabsorption-, ICP-Atomemissions- und ICP-MassenSpektrometrie sowie Ionenchromatografie. Entsprechende Kalibrationsproben können durch geeignetes Mischen von Reinelementlösungen relativ einfach, rasch und in vielfältigen Kombinationen hergestellt werden. Im Gegensatz dazu ist bei der Direktanalyse fester Proben der Zugang zu geeigneten Kalibrationsproben sehr viel schwieriger. Bei exotischen Elementzusammensetzungen sind keinerlei zertifizierte Referenzproben erhältlich.

Praktisch sämtliche Festproben lassen sich nach vorgängiger Zerkleinerung durch geeignete Aufschlussoperationen in Lösung überführen. Dafür stehen in einem gut ausgerüsteten Laboratorium eine Reihe verschiedener Verfahren zur Verfügung. Die übliche Methode bedient sich der Behandlung mit starken Mineralsäuren bei verschiedenen Temperaturen und z.T. unter Druck in Bombenrohren und Autoklaven. Je nach Probenart und den zu bestimmenden Elementen werden Salz-, Salpeter-, Schwefel-, Perchlor-oder Fluss- säure allein oder in verschiedensten Kombinationen und Konzentrationen eingesetzt. Gezielte Zugabe von Oxidationsmitteln wie Peroxiden und Brom, Komplexbildnern und weiteren Reagenzien ermöglichen einen vollständigen Lösevorgang auch für schwierige Proben. Eine besonders vielseitige und leistungsfähige Apparatur stellt das Hochdruckaufschlussgerät (HPA) dar, bei dem die Aufschlussreaktion in geschlossenen Gefässen aus Quarz oder Glaskohlenstoff bei hohen Drucken und Temperaturen ausgeführt wird. Auch Mikrowellenöfen werden eingesetzt. Trockenveraschung und Überführung der Asche in Lösung sowie Schmelzaufschlussoperationen sind weitere Möglichkeiten, eine Probe in Lösung zu überführen.

Der Nachteil des Probenaufschlusses ist neben dem Aufwand für den Reaktionsprozess die Tatsache, dass die Probe dabei verdünnt wird. Der Verdünnungsfaktor liegt generell zwischen ungefähr 10 und 1000. Dies wirkt sich auf das analytische Nachweisvermögen nachteilig aus.

\section{Automatisierung}

Unabdingbare Voraussetzung jeder Instrumentierung und Automatisierung im modernen analytischen Laboratorium ist der Computer. Automatisierung kann in vier Bereichen erfolgen wie in Fig. 11 schematisch dargestellt. Im Bereich Betrieb und Steuerung der Geräte ist die aktuelle Realisierung im analytischen Labor am weitesten fortgeschritten. Das
Gebiet der Implementierung von Methoden und Know-how in Expertensystemen ist noch wenig entwickelt. Es existieren jedoch bereits konkrete Ansätze [23]. In allen Bereichen liegt noch viel Potential für zukünftige Entwicklungen auf dem Weg zur Automatisierung.

\section{Qualität, Qualitätssicherung}

Qualitätssicherung (QS) ist vor dem Hintergrund eines wirtschaftlichen und politischen Zusammenschlusses in Europa und einer generellen internationalen Vereinheitlichung von Normen und Prüfvorschriften von grosser Aktualität. Dieser Trend erfasst primär industrielle und Dienstleistungs-Laboratorien sowie solche mit Überwachungs- und Kontrollaufgaben. Der Trend dürfte sich in Zukunft auch auf rein wissenschaftlich ausgerichtete Institutionen ausdehnen. Die Zielsetzungen der Qualitätssicherung sind

- die Festlegung und Überprüfung, dass im betreffenden Laboratorium Mindestanforderungen erfüllt sind und eingehalten werden; dies ist Voraussetzung für die

- gegenseitige Anerkennung von Analysen- und Untersuchungsresultaten.

Um diese Ziele zu erreichen, müssen sowohl organisatorische wie fachliche Massnahmen realisiert werden. Die Normanforderungen sind gemäss GLP (gute Laborpraxis), EN 45001 oder ISO Guide 25 festgelegt [24]. Dazu gehören, je nach zugrundeliegendem System, Massnahmen zum Betrieb des Laboratoriums, zum Analysenablauf, zur Dokumentierung und 
Fig. 12. Entwicklung der anorg. Instrumentalanalytik
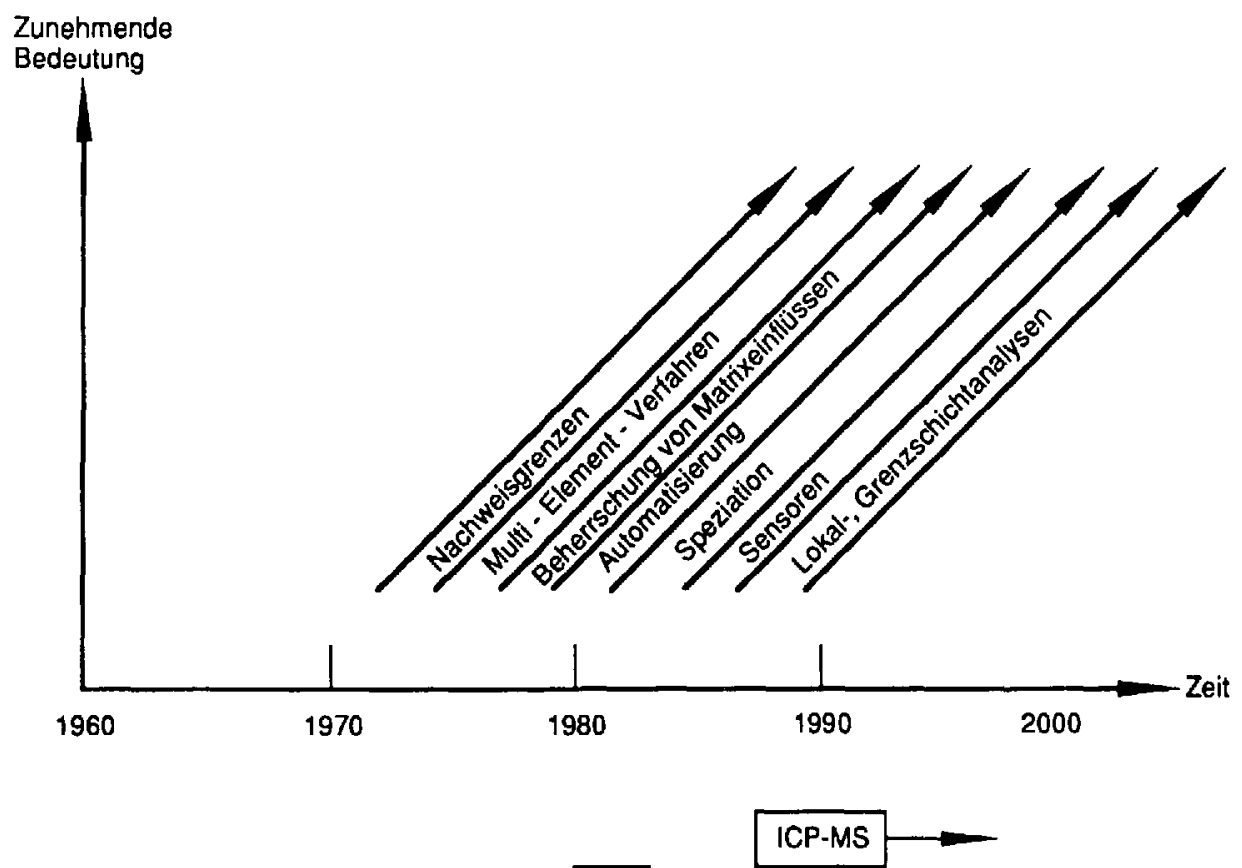

Archivierung, der Gerätewartung und bedienung, Berichterstattung, Teilnahme an Ringversuchen ('proficiency tests'). Auch weitergehende Aspekte wie Qualifikation und Schulung des Personals und Regelung von Sicherheitsfragen sind Teil eines QS-Systems. Interne und externe Kontrollmechanismen und Audits begleiten und unterstützen nicht nur die Systemeinführung, sondern gewährleisten auch dessen Unterhalt. Die Bestätigung der Konformität erfolgt durch Anerkennung nach GLP, Zertifizierung bzw. Akkreditierung durch eine dazu ermächtigte Institution.

Unbedingte Voraussetzung für den Erfolg eines QS-Systems ist die Unterstiutzung durch das oberste Management. Der manifestierte Wille, ein QS-System zu schaffen und zu betreiben, sind in ihrer Tragweite unternehmerische Entscheide. QS-Richtlinien sind das eigentliche Regelwerk des Systems. Der Laborleitung, dem Analytiker obliegen vor allem Massnahmen im fachlichen Bereich wie Erstellung von Arbeitsvorschriften ( $\mathrm{SOP}=$ standard operating procedure), LOG-Büchern für die analytischen Geräte, Prüfplänen und vollständig dokumentierten Analysenabläufen. Die relevanten Dokumente sind im sogenannten Qualitäts-Handbuch vereinigt. Die vollständige Archivierung von den Rohdaten bis zum untersuchten Prüfmaterial stellt sicher, dass die Analyse zu einem späteren Zeitpunkt rückverfolgbar ist.

Die Sicherung der Qualität des eigent- lichen analytischen Resultates an sich ist gerade bei instrumentellen Verfahren äusserst wichtig. Neben einer sorgfältigen Kalibrierung muss eine regelmässige Kontrolle und Wartung der Geräte garantiert und in einem LOG-Buch dokumentiert sein. Die Zuverlässigkeit des analytischen Resultates kann durch verschiedene Massnahmen verbessert werden. Dazu gehören die gleichzeitige Analyse von durch Elementaddition aufbereiteten Untersuchungsproben zusammen mit der Probe oder von Kontrollproben und Referenzmustern mit bekannten, zertifizierten Elementmassenanteilen. Eine Vergleichsanalyse im eigenen Laboratorium mit einer grundsätzlich verschiedenen Methode hilft systematische Fehler zu vermeiden. Regelmässige Teilnahme an Ringversuchen gemeinsam mit anderen Laboratorien auf nationaler und internationaler Ebene sind unerlässliche Massnahmen zur Qualitätssicherung.

\section{Bemerkung über Speziation}

Zusätzlich zur Quantifizierung des Elementgehaltes wird immer häufiger eine Charakterisierung der relativen Anteile nach chemischer Bindungsform notwendig. Kombinierte Analysenverfahren eröffnen in dieser Hinsicht neue Möglichkeiten.

Neben Ionenchromatografie und anderen chromatografischen Techniken sind eine Reihe weiterer Methoden zu nennen.
Dazu gehören namentlich die IR- und Ramanspektroskopie, die Kernresonanzspektroskopie, die Thermogravimetrie, weiter Lichtmikroskopie sowie die $\mathrm{Ra}$ sterelektronenmikroskopie (REM).

Mit der Röntgendiffraktion (XRD) schliesslich werden kristalline Phasen qualitativ und quantitativ erfasst und entsprechenden Kristallarten zugeordnet, was bei parallel dazu ausgeführter Spektralanalyse eine äusserst informative Charakterisierung unbekannter Probenmaterialien erlaubt.

Eine spezifische und etwas unkonventionelle, jedoch interessante Anwendung der Graphitrohrtechnik in Kombination mit ICP-MS dient der spezifischen Unterscheidung von anorg. und org. gebundenem Chlor im Spurenbereich im Zusammenhang mit der Prüfung auf polychlorierte Biphenyle (PCB) [25]. Das temperaturgesteuerte Graphitrohr für die fraktionierte Verflüchtigung der verschiedenen Chlorverbindungen und das nachgeschaltete ICP-MS als Anregungs- und zeitaufgelöstes Detektionssystem funktionieren dabei als Tandem.

\section{Entwicklung der anorganischen In- strumentalanalytik, Trends}

Die historische Entwicklung des Begriffes 'Spuren' veranschaulicht deutlich einen massgebenden Trend der Analytik, nämlich die Entwicklung zu immer tieferen Nachweisgrenzen. Anfangs unseres 
Jahrhunderts wurde mit dem Begriff 'Spuren' ein Elementmassenanteil von ca. $0,1 \%$ verstanden, 1960 waren es ca. $0,01 \%$ und heute viel eher 0,001 oder $0,0001 \%$, bzw. $1 \mathrm{ppm}$. Die permanente Forderung nach tieferen Nachweisgrenzen hat besonders während der letzten zwei Jahrzehnte sowohl den analytischen Chemiker als auch die Instrumentenhersteller herausgefordert. Der Übergang von Einzelelementbestimmungen zu Multielementmethoden, der zunehmende Grad der Automatisierung sowie die Beherrschung von Matrixeinfluissen bedeuteten weitere wichtige Entwicklungen. In Fig. 12 ist dies schematisch dargestellt. Die gesteigerte Leistungsfähigkeit in der anorg. Elementanalytik wurde instrumentell ermöglicht durch Einführung der Atomabsorption in den 60er Jahren, durch die Renaissance der AES mit Einführung des ICP, Weiterentwicklung der Röntgenfluoreszenz und der flammenlosen Atomabsorption in den 70er und 80er Jahren und schliesslich durch Einführung der Ionenchromatografie und die gegenwärtige rasche Verbreitung der ICP-MS.

Die Zukunft stellt an die Analytik nicht minder anspruchsvolle Forderungen. Dazu gehört die konsequente Fortführung der Automatisierung. Vermehrt gelangen Sensoren (Elektroden und Optroden) für eine analytische Überwachung an Ort und Stelle zum Einsatz [26]. Dadurch entfallen Probenahme und Überführung ins analytische Labor. Zunehmende Bedeutung erhalten Lokalanalysen im mikroskopischen und submikroskopischen Bereich, insbesondere in der Werkstofforschung, der Elektronik und der Umweltanalytik.

Der aktuelle Stand der Technik zeigt, dass eine Vielzahl leistungsfähiger Methoden für die instrumentelle anorg. Analytik existiert und in gut ausgerüsteteten analytischen Laboratorien etabliert ist. Eine Reihe von Verfahren arbeiten nach denselben Grundprinzipien, unterschiedlich ist jedoch die analytische Aussagekraft. Die ideale Methode, welche alle anderen ersetzen könnte, gibt es (noch?) nicht. Vielmehr sind die gängigen Verfahren in vielerlei Hinsicht komplementär. Galt die wellenlängendispersive RFA während längerer Zeit als relativ universelle Elementbestimmungsmethode, trifft dies heute und in noch ausgeprägterem Mass für die mit diversen Optionen ausgerüstete ICP-MS zu.

Trotz der dynamischen Entwicklung der Instrumentalanalytik und dem an sich wünschbaren Trend zu Computerisierung und Automatisierung kommerzieller Instrumente bleibt der Mensch, der erfahrene chemische Analytiker nach wie vor der entscheidende Faktor. Er ist fähig, ein neues analytisches Verfahren zu entwik- keln, zur Lösung einer Problemstellung die geeignete Methode zu wählen und ein instrumentelles Verfahren optimal einzusetzen und zu betreiben. Er ist es, der kritisch, sachlich und problemorientiert die Qualität der enormen Fülle an Resultaten begutachtet und beurteilt. Daran wird sich so schnell nichts entscheidendes ändern.

Bei dieser Arbeit handelt es sich um eine überarbeitete Fassung eines Vortrags 'Moderne Instrumentalanalytik für anorg. Stoffe in Wasser und Feststoffen', gehalten am 30.8.1991 an der 28. Chemikertagung des Vereins Schweizerischer Abwasserfachleute, VSA in Zürich. Sie wäre in dieser Form nicht zustandegekommen ohne die vielen wertvollen Anregungen und kritischen Diskussionen, die ich insbesondere J. Zobrist, EAWAG, CH-8600 Dübendorf und $P$. Lienemann, EMPA herzlich verdanke. Ebenso gilt mein grosser Dank Frau $M$. Boll und $P$. Boll für den engagierten Einsatz bei der Verfassung des Manuskriptes, $H$. Schallert für die Umsetzung von Figuren.

\section{Eingegangen am 24. Januar 1992}

[1] B.L. Karger, 'Recent trends in analytical biotechnology', Schweiz. Chem. Gesellschaft, Herbstversammlung 18.10.91 in Bern.

[2] M. Grasserbauer, Fresenius J. Anal. Chem. 1991, 341, A9; H.M. Widmer, B. Weiss, A. Fiechter, Chimia 1991, 45, 391.

[3] G. Kirchhoff, R. Bunsen, Ann. Phys. Chem. 1860, 110,161 .

[4] G. Tölg, GIT Fachz. Lab. 1989, 10, 971; G.M. Hieftje, Fresenius J. Anal. Chem. 1990, 337, 528; B.V. L'vov, Anal. Chem. 1991, 63, 924A.

[5] T. Braun, Fresenius Z. Anal. Chem. 1987, $328,1$.

[6] B.V. L'vov, W. Slavin, J. Anal. At. Spectr. 1991, 6,191 .

[7] T.B. Reed, J. Appl. Phys, 1961, 32, 821 R.H. Wendt, V.A. Fassel, Anal. Chem. 1965 , 37, 920; M. Thompson, J.N. Walsh, 'A Handbook of Inductively Coupled Plasma Spectrometry', Blackie, Glasgow - London, 1983; P. Lienemann, 'Chemisch-physikalische Untersuchung der Atomisierungsprozesse bei Verwendung eines induktiv gekoppelten Argonplasmas in der optischen Atomspektrometrie', Diss. ETH Nr. 8195, Zürich, 1986; N. Kläntschi, A. Esenwein, T. Müller, Fresenius Z. Anal. Chem. 1987, 328,657 ; H. Vonmont, N. Kläntschi, N. Heeb, '4. Coll. Atomspektrometrische Spurenanalytik', Ed. B. Welz, 1987, S 261 ; Z. Zhanxia, R.B. Baer, V.A. Fassel, Spectrochim. Acta. Sect. B 1991, 46, 1171 ; J.W. Olesik, Anal. Chem. 1991, 63, 12 A.

[8] N. Kläntschi, Mater. Technik 1980, 8, 14.
[9] M.W. Routh, W. Vogel, F. Pilloud, P. Cassagne, D.F. Sermin, R.C. Fry, B. Kennessey, XXVII Colloquium Spectroscopicum Internationale 1991, A-2.2.

[10] T.H. Bandemer, Int. Lab. 1990, (Oct) 26.

[11] G. Horlick, S.H. Tan, M.A. Vaughan, Y. Shao, "Inductively coupled plasma-mass spectrometry', VCH, Weinheim, 1987; A.R. Date, A.L. Gray, 'Applications of ICPMS', Blackie, Glasgow - London, 1989; D.J. Douglas, Can. J. Spectrose, 1989, 34, 38; D. Ekimoff, A.M. van Norstrand, D.A. Mowers, Appl. Spectrosc: 1989, 34, 1252; K.E. Jarvis, J. Anal. At. Spectrosc. 1989, 4, 563; E.R. Denoyer, K.J. Fredeen, J.W. Hager, Anal. Chem. 1991, 63, 445A.

[12] J. Stummeyer, G. Wünsch, Fresenius J. Anal. Chem. 1991, 340, 269.

[13] A.Tsumura, S. Yamasaki, 'Applications of Plasma Source Mass Spectrometry', Eds. G. Holland and A.N. Eaton, The Royal Society of Chemistry, Cambridge, 1991, S. 119.

[14] E.P. Bertin, 'Principles and Practice of XRay Spectrometric Analysis', Plenum Press New York, 1975; R. Klockenkämper, Spectrochim. Acta, Sect. B 1987, 42, 423.

[15] A. Prange, GIT Fachz. Lab. 1987, 6, 513; R. Klockenkämper, Spectrosc. Int. 1990, 2 , 26; P. Boumans, P. Wobrauschek, H. Aiginger, Eds., Proceedings of the Third Workshop on Total Reflection X-Ray Fluroescence (TXRF), Vienna, Austria 15-17 May 1990, Spectrochim. Acta, Sect. B 1991, 46, 1313-1436.

[16] U. Bilitewski, T. Ding, R.D. Schmid, Angew. Chem. 1991, 103, A-107; K. Bächmann, I. Haag, U. Sprenger, K.H. Steeg, K. Steigerwald, B. Bastian, A. Röder, Fresenius J. Anal. Chem. 1991, 340, 548.

[17] M. Grasserbauer, Trends Anal. Chem. 1989. 8, 192; M. Grasserbauer, Phil. Trans. $R$. Soc. London 1990, A333, I13; J.C. Riviere, 'Surface Analytical Techniques', Clarendon Press, Oxford 1990, H. Pulm, GIT Fachz Lab. 1991, 91, 969.

[18] K. Niemax, J. Anal. Chem. 1990, 337, 55!; G. Tölg, Fresenius J. Anal. Chem. 1991, 339,451 .

[19] W. Slavin, Spectrosc. Int. 1992, 4, 22.

[20] D.L. Massart, B.C.M. Vandenginste, S.M. Deming, Y. Michotte, L. Kaufman, 'Chemometrics: a textbook', Elsevier, Amsterdam, 1988; K. Ohls, D. Sommer, Fresenius. Z. Anal. Chem. 1982, 312, 195; H.S. Hertz, Anal. Chem. 1988, 60, 75A.

[21] N. Kläntschi, ICP Inform. Newslett. 1991, I6, 733 .

[22] C.M. Beck, Anal. Chem. 1991, 63, 993A; J.R. Moody, M.S. Epstein, Spectrochim. Acta, Sect. B 1991, 46, 1571 .

[23] S. Lahiri, M.J.Stillman, Anal. Chen. 1992, $64,283 \mathrm{~A}$.

[24] K. Heydorn, ibid. 1991, III, I; W. Merz, R. Wittlinger, ibid. 1991, III, II; B.E. Broderick, Mikrochim. Acfa 1991, $I I I, 17$

[25] P. Richner, S. Wunderli, FAACS1 Pacific Conference, 27th Western Regional ACS Meeting, Anaheim, CA, 1991 , paper 676.

[26] H.M. Widmer, Chimia 1987, 4l, 432. 